\title{
Two Retinotopic Visual Areas in Human Lateral Occipital Cortex
}

\author{
Jonas Larsson and David J. Heeger \\ Department of Psychology and Center for Neural Science, New York University, New York, New York 10003
}

We describe two visual field maps, lateral occipital areas 1 (L01) and 2 (LO2), in the human lateral occipital cortex between the dorsal part of visual area $\mathrm{V} 3$ and visual area V5/MT + . Each map contained a topographic representation of the contralateral visual hemifield. The eccentricity representations were shared with $\mathrm{V} 1 / \mathrm{V} 2 / \mathrm{V} 3$. The polar angle representation in $\mathrm{LO} 1$ extended from the lower vertical meridian (at the boundary with dorsal V3) through the horizontal to the upper vertical meridian (at the boundary with L02). The polar angle representation in L02 was the mirror-reversal of that in L01. L01 and L02 overlapped with the posterior part of the object-selective lateral occipital complex and the kinetic occipital region (KO). The retinotopy and functional properties of LO1 and LO2 suggest that they correspond to two new human visual areas, which lack exact homologues in macaque visual cortex. The topography, stimulus selectivity, and anatomical location of LO1 and LO2 indicate that they integrate shape information from multiple visual submodalities in retinotopic coordinates.

Key words: fMRI; homology; human; lateral occipital; retinotopy; V4; visual cortex

\section{Introduction}

The primate visual cortex can be subdivided into a number of distinct areas on the basis of retinotopy, functional properties, anatomical connections, and microstructure (Gattass et al., 2005). Many of these areas have been described only in nonhuman primates (particularly the macaque), but a growing number have also been identified in humans (Zeki et al., 1991; Watson et al., 1993; Engel et al., 1994; Sereno et al., 1995; Tootell et al., 1995, 1997, 1998; DeYoe et al., 1996; Press et al., 2001; Huk et al., 2002; Brewer et al., 2005). Several homologous visual areas can be identified in both species, including V1, V2, V3, V3A, and V5/MT+. Beyond V3, however, the homology between human and monkey areas is less certain (Tootell et al., 1997; Smith et al., 1998; Press et al., 2001; Tootell and Hadjikhani, 2001; Brewer et al., 2005; Schluppeck et al., 2005; Silver et al., 2005).

The retinotopic organization in the human lateral occipital cortex between dorsal V3 and V5/MT+ remains incompletely characterized. A few studies have reported that this region of cortex exhibits an eccentricity bias, but lacks a clear map of polar angle (Levy et al., 2001; Tootell and Hadjikhani, 2001; Tyler et al., 2005b). Functional studies have shown that this region is part of the object-selective lateral occipital complex (LOC) (Malach et al., 1995; Grill-Spector et al., 1998a; Levy et al., 2001; Hasson et al., 2003; Kourtzi et al., 2003) and the kinetic occipital region (KO) (Dupont et al., 1997; Van Oostende et al., 1997). Both LOC

Received April 18, 2006; revised 0ct. 3, 2006; accepted Oct. 4, 2006.

This work was supported by National Eye Institute Grants R01-EY11794 (D.J.H.) and R01-EY16165 (Michael Landy) and a grant from the Seaver Foundation. We thank Alyssa Brewer, Michael Landy, Tony Movshon, Andy Smith, Brian Wandell, and members of the Heeger laboratory for discussion and comments.

Correspondence should be addressed to Jonas Larsson, Department of Psychology and Center for Neural Science, New York University, New York, NY 10003. E-mail: jonas@cns.nyu.edu.

DOI:10.1523/JNEUROSCI.1657-06.2006

Copyright $\odot 2006$ Society for Neuroscience ～0270-6474/06/2613128-15\$15.00/0 and $\mathrm{KO}$ are large and functionally heterogeneous cortical regions that are believed to include multiple subregions (Grill-Spector et al., 1999; Hasson et al., 2003; Zeki et al., 2003; Sawamura et al., 2005; Tyler et al., 2005a). In the macaque there are at least two distinct visual areas between dorsal V3 and MT: dorsal V4 (V4d) (Zeki, 1971, 1977; Gattass et al., 1988; Brewer et al., 2002; Fize et al., 2003) and an area that has been called either V4t (Desimone and Ungerleider, 1986) or MTc (Tootell and Taylor, 1995).

We used functional magnetic resonance imaging (fMRI) to study the retinotopic organization of human lateral occipital cortex. Our data suggest that there are two adjacent retinotopic maps of the contralateral hemifield in the region between dorsal V3 (V3d) and V5/MT +. In contrast with previous reports, we found that these maps contained orderly representations of both visual field eccentricity and visual polar angle. Unlike macaque V4d and V4t, which represent only the lower quadrant, our results indicated that the two human maps in this region each contained a full hemifield representation, implying that they are not directly homologous to the macaque areas. We propose that these maps are two new human visual areas, which we have labeled LO1 and LO2 (for lateral occipital areas 1 and 2). The functional properties of LO1 and LO2 suggest they play different but complementary roles in shape recognition. Some of these results have been published previously in preliminary form (Larsson et al., 2006).

\section{Materials and Methods}

\section{Subjects and scanning sessions}

Fifteen experienced subjects participated in this study with written consent. Procedures complied with safety guidelines for MRI research and were approved by the human subjects Institutional Review Board at New York University. Each subject participated in at least two scanning sessions: one to obtain a high-resolution anatomical volume, and another to measure the retinotopic maps in visual cortex (see below). Nine subjects participated in an additional session to localize V5/MT+ and, in seven of 
these subjects, we also localized LOC and KO in the same session. Three subjects participated in a previous study that measured orientationselective adaptation in the visual cortex (Larsson et al., 2006).

\section{Cortical surface extraction and analysis}

Inner (gray/white) and outer (pial) cortical surfaces were extracted from high-resolution T1-weighted MR images [magnetization-prepared rapid-acquisition gradient echo (MPRAGE); voxel size $1 \times 1 \times 1 \mathrm{~mm}$ ] of each subject using the public domain software SurfRelax (Larsson, 2001) (www.cma.mgh.harvard.edu/iatr). Surface patches covering the occipital cortex (roughly defined as the cortex within $10 \mathrm{~cm}$ surface distance from the occipital pole) of each hemisphere were cut from the inner cortical surface and flattened for visualization of functional data. Calculations of surface area and distance were done on the triangulated mesh representation of the original, folded surface. Mesh distances (number of edges) were measured along the folded white matter surface using a flood-fill algorithm. The distances calculated by this procedure are equivalent to shortest path distances between pairs of vertices obtained by Dijkstra's algorithm (Dijkstra, 1959). Mesh distances were converted to millimeters by scaling with the average edge length, divided by a heuristic correction factor of 1.2. This factor compensated for the overestimation of distances that result from approximating a smooth surface by a discrete triangulated mesh. The correction factor was obtained by repeatedly measuring the mesh distance between random pairs of vertices on synthetic triangulated parabolic surface patches and dividing this distance by the true distance (calculated analytically as the length of the line integral between the two vertex coordinates). The correction factor was essentially constant for parabolic surfaces with a radius of curvature $8 \mathrm{~mm}$ or larger (similar to the curvature of cortical surfaces). Although there exist more exact methods for estimating geodesic distances along triangulated manifolds (Kimmel and Sethian, 1998), we chose this heuristic for its simplicity. Because we averaged our distance measurements across many vertices and subjects, the mean error in our distance measurements is likely to be small.

\section{fMRI methods}

Data were acquired on a Siemens (Erlangen, Germany) 3T Allegra scanner equipped with a transmit head-coil (NM-011; NOVA Medical, Wakefield, MA) and a four-channel phased array receive coil (NMSC021; NOVA Medical). Echoplanar imaging was used to measure blood oxygenation level-dependent (BOLD) changes in image intensity (Ogawa et al., 1990). Parameters for the retinotopic mapping scans were as follows: 24 slices (21 in one subject); repetition time (TR) $=1500 \mathrm{~ms}$ (2400 in one subject); echo time (TE) $=30 \mathrm{~ms}$; flip angle $=75^{\circ}\left(90^{\circ}\right.$ in one subject); $64 \times 64$ matrix size; voxel size, $3 \times 3 \times 3 \mathrm{~mm}$. The same parameters were used for the V5/MT + , LOC, and KO localizer scans. For one subject, retinotopic mapping scans were performed on a full-body General Electric (Milwaukee, WI) 3T scanner equipped with a custommade two-channel surface coil using a two-shot spiral acquisition sequence $(\mathrm{TR}=1200 \mathrm{~s}$; $\mathrm{TE}=30 \mathrm{~s}$; voxel size, $2.5 \times 2.5 \times 3 \mathrm{~mm})$. In the orientation-selective adaptation scans, described in detail previously (Larsson et al., 2006), we used fewer slices (19) and a shorter TR (1200 $\mathrm{ms})$, but otherwise similar parameters.

Data from each scanning session were coregistered. At the beginning of each session, we acquired a T1-weighted (MPRAGE) anatomical volume in the same slices as the functional scans, but with twice the in-plane resolution (voxel size, $1.5 \times 1.5 \times 3 \mathrm{~mm}$ ). This anatomical volume was aligned with the subject's high-resolution anatomical scan (used for cortical surface extraction) (see above) by an automated robust image registration algorithm (Nestares and Heeger, 2000). The alignment parameters were used to project the measured fMRI responses onto the flattened cortical surfaces for visualization.

The data from each functional scan were preprocessed using standard procedures for motion compensation (Jenkinson et al., 2002) and detrending (Zarahn et al., 1997; Purdon and Weisskoff, 1998), and then analyzed by fitting a sinusoid to the time series at each voxel and computing (1) the correlation (technically coherence) between the measured time series and the best-fitting sinusoid (Bandettini et al., 1993; Engel et al., 1997), and (2) the response phase (Engel et al., 1994). For the retino- topic mapping experiments, response phase corresponded to visual field location. For the localizer experiments, response phase reflected the stimulus condition that evoked the larger response (e.g., objects vs scrambled objects).

\section{Retinotopic mapping}

Retinotopic visual areas were identified by measuring the BOLD fMRI response in the visual cortex of human observers viewing high-contrast checkerboard stimuli that gradually traversed the visual field (Engel et al., 1994). The polar angle components of the retinotopic maps were measured using stimuli restricted to a wedge that rotated (clockwise or counter-clockwise) slowly around the fixation point. The radial components were measured using stimuli restricted to a ring that gradually expanded from or contracted toward the fixation point. These stimuli evoked traveling waves of activity simultaneously in multiple retinotopically organized visual cortical areas. The temporal phases of the fMRI responses in each voxel indicated the corresponding visual field location. The resulting retinotopic maps were visualized on flattened surface representations of each subject's occipital cortex.

Visual stimuli were presented at $800 \times 600$-pixel resolution with a refresh rate of $60 \mathrm{~Hz}$ on an electromagnetically shielded analog NEC2110 liquid crystal display (LCD). The display was located behind the scanner bore at a viewing distance of $150 \mathrm{~cm}$, yielding a viewing angle of $\sim 16 \times$ $12^{\circ}$. (For the one subject scanned with a GE scanner, viewing distance was $320 \mathrm{~cm}$, yielding a smaller viewing angle.) Stimuli consisted of a black and white high-contrast radial checkerboard pattern presented within a slowly rotating (clockwise or counter-clockwise, in stepwise increments of 15 angular degrees per TR) wedge aperture or a slowly expanding or contracting (in stepwise increments of $0.375^{\circ}$ eccentricity per TR) ring aperture. Each radial strip of the checkerboard pattern moved randomly inward or outward on each stimulus frame at a speed of $2 \%$, giving rise to vivid motion boundaries between adjacent strips. Wedge apertures subtended $45^{\circ}$ of polar angle and extended from 0.4 to $5.8^{\circ}$ eccentricity. The relatively narrow width of the wedge apertures was chosen to evoke stronger modulation in areas with large response fields (Tootell et al., 1997). Ring apertures subtended $1.2^{\circ}$ eccentricity and traversed the same range of eccentricities as the wedge apertures $\left(0.4-5.8^{\circ}\right)$. Regions outside the wedge or ring apertures were a uniform gray. Each $252 \mathrm{~s} \mathrm{scan}$ consisted of 10.5 cycles (rotations or expansions/contractions); the first half cycle of each scan was discarded before analysis. For each subject, we ran six scans with wedge stimuli (three in each direction) and four scans with ring stimuli (two in each direction). The counter-clockwise rotating wedge stimulus sequence was the exact reverse of the clockwise stimulus sequence (after removal of the first half cycle), and likewise for the expanding and contracting ring stimuli.

Data acquired with different stimulus directions were combined to estimate the response phase independent of the phase lag caused by the hemodynamic delay of the fMRI response. Time series data for each scan were first coarsely corrected for hemodynamic delay by shifting the time series of each voxel back three time points (corresponding to $4.5 \mathrm{~s}$ ). The time series for the counter-clockwise wedge and contracting rings were then time-reversed and averaged with the time series data for clockwise wedges and expanding rings, respectively. This way, any residual phase lag was canceled, allowing us to directly convert response phase into polar angle or eccentricity without having to estimate hemodynamic delay.

Retinotopic visual area boundaries were identified in the flattened retinotopic maps using four criteria (Engel et al., 1994; Sereno et al., 1995; DeYoe et al., 1996; Engel et al., 1997). (1) The response phases progressed across each visual area indicating a topographic organization of the visual field on the cortical surface. (2) There were phase reversals in the polar angle components of the retinotopic maps, located at boundaries between adjacent visual areas sharing a common eccentricity representation. The polar angle maps in adjacent visual areas were mirror images of each other, so the phase progressions were in opposite directions along the cortical surface, with a phase reversal along their shared boundary. (3) Alternatively, adjacent areas shared a common polar angle representation but had separate eccentricity representations that extended in opposite directions from a shared foveal representation. This 
criterion was used specifically to define the boundary between V3A and V3B (Wandell et al., 2005). (4) The angular and radial components within each visual area were either nearly orthogonal to one another or consistently skewed, but not parallel to one another.

\section{Cortical magnification}

We calculated cortical magnification functions for V1, V2, V3, hV4, LO1, and $\mathrm{LO} 2$ in the nine subjects with the clearest retinotopy in LO1 and LO2. We measured the distance from the foveal representation to each location in the folded cortical surface. For each visual area, we sorted the estimated distances into 10 evenly spaced distance bins and averaged the eccentricities within each bin. Data for different subjects were aligned by shifting the distances for each visual area to a common origin, corresponding to the $3^{\circ}$ isoeccentricity contour. We included voxels that responded with coherence values 0.5 or greater to the expanding or contracting ring stimuli. Using a lower coherence threshold (0.25) resulted in increased spread of data points, but did not substantially change the shape of the plots or the estimated cortical magnification functions. Data from 1 to $5^{\circ}$ eccentricity were fit with functions of the form $E=\exp [a(D$ $+b)$ ] to the data, where $E$ is eccentricity in degrees, $D$ is cortical distance in millimeters, and $a$ and $b$ are constants.

\section{Voxel response field sizes}

Voxel response fields (the region of visual space evoking a response in a voxel) were estimated for all voxels showing a strong response modulation (defined as voxels having a response coherence $>0.25$ ). Response fields were modeled as two-dimensional (2D) Gaussian functions in polar coordinates, centered on the visual field location (eccentricity and polar angle) measured for each voxel. By modeling the response fields in polar coordinates rather than in Cartesian coordinates, we could separately estimate response field size in the radial (eccentricity) and polar dimensions. For each voxel, the individual (detrended and zero mean normalized, but not shifted or reversed in time) time series for the four different stimulus conditions (clockwise and counterclockwise rotating wedges, expanding and contracting rings) were averaged across scan repeats (three repeats for wedge stimuli, two repeats for ring stimuli). The response field size for each voxel was estimated by generating simulated time series for each of the four stimulus conditions, varying the response field size parameters to minimize the difference between the measured and simulated time series. For each stimulus condition, simulated time series were generated by computing the inner product between the response field (modeled as 2D image matrices) and stimulus contrast images representing the visual stimulus at each time frame. The stimulus contrast images had a value of 1 (full contrast) at wedge or ring stimulus locations and a value of 0 (zero contrast) elsewhere. The resulting time series were convolved with the hemodynamic impulse response function of SPM99 software (Wellcome Department of Cognitive Neurology, London, UK; http://www.fil.ion.ucl.ac.uk/spm) with default parameters (response delay, $6 \mathrm{~s}$; undershoot delay, $16 \mathrm{~s}$; dispersion of response and undershoot, $1 \mathrm{~s}$; ratio of response to undershoot, 6) to generate a simulated fMRI response time series. Both simulated and measured time series were normalized to zero mean and an SD of 1 . Response field parameters were estimated by nonlinear least-squares minimization (Levenberg-Marquardt) of the mean squared difference between the simulated and measured time series simultaneously for all four stimulus conditions. To constrain the fit, response field sizes were fit in separate steps for the polar and radial dimensions (in that order), with the other parameter held fixed. Initially, we also performed a final simultaneous fit of both parameters, but this additional step did not improve the fit and was therefore excluded. The fitting procedure yielded, for each voxel, an estimate of response field size expressed as the SDs of the fitted Gaussian functions in the polar and radial dimensions. For the scatter plots in Figure 6, response field sizes in the polar dimension (in radians) were converted to degrees of visual angle by scaling by the eccentricity of each voxel. We included only voxels with eccentricities $>0.5$ and $<3.5^{\circ}$; the fitting procedure often failed to converge for voxels outside this eccentricity range because of weaker response modulation near the fixation point and the outer edge of the stimulus.

\section{Visual field coverage}

The visual field coverage (i.e., the region of visual space represented in a visual area), was estimated for individual visual areas by summing the voxel response fields of all voxels showing strong response modulation (coherence $>0.25$ ). Specifically, the response fields for individual voxels were thresholded at the full width at half maximum value and summed across voxels, separately for each visual area and subject. The contours in Figure 7 correspond to half maximum values of the summed response fields obtained by this procedure.

\section{Retinotopic atlas fitting procedure}

We fit a quantitative model of visual field topography, which we refer to as an atlas, to data from individual subjects using an automated elastic deformation algorithm (Dougherty et al., 2003) (for details, see supplemental Fig. 5, available at www.jneurosci.org as supplemental material). The atlas consisted of templates of the expected polar angle and eccentricity maps of these areas, as inferred from our observations (for LO1 and LO2) and previous retinotopic mapping studies for V1, V2, V3 (Engel et al., 1994; Sereno et al., 1995; Tootell et al., 1995; DeYoe et al., 1996), hV4 (Brewer et al., 2005; Wandell et al., 2005), and V3A/B (Tootell et al., 1997, 1998; Press et al., 2001; Wandell et al., 2005). Importantly, the fitting algorithm enforced the constraint that eccentricity and polar angle were represented independently (i.e., not parallel), and also helped reduce observer bias in locating area boundaries in the flat maps. The templates were defined in an arbitrary atlas coordinate system as rectangular matrices (arbitrarily defining a full hemifield as $100 \times 100$ pixels) representing the polar angle and eccentricity maps in each area. Eccentricity in the template maps increased along the ordinate from 1 to $6^{\circ}$. The eccentricity range in the template maps for $\mathrm{LO} 1$ and $\mathrm{LO} 2$ was restricted between 1 and $4.5^{\circ}$, reflecting the smaller range of eccentricity representation observed in these areas (see Results). Polar angle increased or decreased (depending on area) along the abscissa in the template maps between $-\pi / 2$ and $\pi / 2$ (for V1, hV4, V3A/B, LO1, and LO2) or between $\pm \pi / 2$ and 0 (for V2v, V2d, V3v, and V3d) (supplemental Fig. $5 A, B$, available at www.jneurosci.org as supplemental material). Note that our definition of hV4 followed that of Brewer et al. (Brewer et al., 2005), identifying this area as a full hemifield representation directly anterior to $\mathrm{V} 3 \mathrm{v}$.

The outer meridian boundaries of V3A/B (with V7), hV4 (with visual areas $\mathrm{VO} 1$ and/or TEO), and LO2 (with V5/MT+) were associated with a phase reversal in the polar angle maps. To ensure that the fitting algorithm aligned the outer boundaries of these areas without having to explicitly include the neighboring areas in the atlas, the outer borders of the templates for $\mathrm{V} 3 \mathrm{~A} / \mathrm{B}, \mathrm{hV} 4$, and $\mathrm{LO} 2$ were padded with dummy polar angle template maps each representing a phase-reversed quadrant (supplemental Fig. 5C, available at www.jneurosci.org as supplemental material). Because the eccentricity representations in these neighboring areas (V7, VO1, and V5/MT+) were often indistinct, we did not fit eccentricity templates to these areas. For the same reason, only polar angle templates were used for $\mathrm{V} 3 \mathrm{~A} / \mathrm{B}$ (compare the eccentricity and polar angle template maps in supplemental Figs. $5 C, D$, available at www. jneurosci.org as supplemental material).

For each hemisphere, the boundaries of the template maps were first manually coarsely aligned with the corresponding polar angle reversals in the flat maps (supplemental Fig. 5C,D, available at www.jneurosci.org as supplemental material). The elastic deformation algorithm then iteratively warped the template maps to minimize the deviation between the templates and the data, simultaneously for polar angle and eccentricity, subject to elasticity (stiffness and skew) constraints (Dougherty et al., 2003) (supplemental Figs. 5E, F, available at www.jneurosci.org as supplemental material). The deformation parameters were set to simulate a relatively stiff, rubber-like material to avoid overfitting the data. The fitting procedure ran for 100-150 iterations until the residual error began to asymptote. Overall, the boundaries identified by the atlas fitting procedure corresponded well to those drawn by manual inspection, and the area estimates obtained by the two methods were very similar.

The average polar angle and eccentricity maps (see Figs. 1-3) were generated by averaging the fMRI phase measurements across subjects in the atlas space. For each area and hemisphere, the algorithm yielded a 
mapping from the individual flat map data to the atlas template space and vice versa. The raw data from individual hemispheres were warped to the atlas space and averaged (see Fig. 8). We then averaged the coordinate transformations (between atlas and flat map) of each area across subjects and, using these average coordinates, warped the averaged polar angle and eccentricity data back to the flat map coordinate system.

\section{Localizer scan protocols}

Visual stimuli for the localizer scans were presented using an LCD projector (Eiki LC-XG100) outfitted with a custom lens (5.2-8.7 inch Xtra Bright; Navatar/Buhl, Rochester NY) on a rear projection screen at $640 \times$ 480 pixel resolution and $60 \mathrm{~Hz}$. Viewing distance was $\sim 60 \mathrm{~cm}$, yielding a viewing angle of $\sim 30 \times 20^{\circ}$.

V5/MT+. A standard motion localizer stimulus (Watson et al., 1993; Tootell et al., 1995; Huk et al., 2002) was used to functionally identify area $\mathrm{V} 5 / \mathrm{MT}+$ in 11 subjects. The stimulus alternated between $12 \mathrm{~s}$ epochs of stationary random dots and $12 \mathrm{~s}$ epochs of moving random dots. The dot patterns consisted of $\sim 1000$ single pixel white dots presented on a black background within a circular aperture with a radius of $10^{\circ}$. During stationary epochs, a different static dot pattern was displayed every $0.5 \mathrm{~s}$. During motion epochs, a different moving dot pattern was shown every $0.5 \mathrm{~s}$. Moving dot patterns were generated by displacing the dots in each pattern in random directions at a constant speed of $5.8^{\circ} \%$ s. The direction of movement of individual dots remained constant throughout each $0.5 \mathrm{~s}$ interval. Subjects underwent two motion localizer scans, each consisting of 10 alternations between stationary and moving epochs.

Lateral occipital complex. The LOC was identified in separate scans in seven subjects. The stimuli consisted of $12 \mathrm{~s}$ sequences of grayscale images of faces and common objects, alternating with $12 \mathrm{~s}$ sequences of scrambled versions of the same images. Individual images were displayed for $0.25 \mathrm{~s}$ each, in random order. Objects and faces were shown in separate, alternating sequences, allowing us to subdivide the LOC into objectpreferring and face-preferring regions. Two or three scans were run per subject, each scan comprising a total of 10 alternations between intact images and scrambled images, corresponding to five cycles of object images and five cycles of face images.

Computer-rendered three-dimensional images of 80 common objects were obtained from the Object DataBank (courtesy of Michael J. Tarr, Brown University, Providence, RI). We restricted the images to convex objects with small aspect ratios to approximately match the size of object and face images (mean size, $\sim 6^{\circ}$ ). For most objects, multiple canonical views of the object were used. Face images ( 40 different faces, each shown in 10 different frontal views) were obtained from the Olivetti Research Laboratory face database (Samaria and Harter, 1994). Both sets of images were converted to grayscale and shown on a uniform gray background.

Scrambled images were generated by subdividing each image into a set of circular cells organized in a hexagonal grid with cell centers spaced 25 pixels apart. An "intensity metric" was computed for each cell as the weighted average of the normalized variance and mean intensity of all pixels inside the cell (variance weight, 0.25 ; mean weight, 0.75 ). The intensity metrics for all cells were sorted into 20 equally sized bins. A scrambled image was generated by randomly permuting the location of the cells within each intensity bin. To avoid introducing intensity edges between adjacent cells, pixel values within each cell were scaled with a Gaussian aperture function that extended into neighboring cells. The resulting scrambled images preserved the local image structure and overall intensity distribution of the original images, but lacked any recognizable global shape. We eliminated images with highly recognizable global shape (e.g., a bicycle) or highly distinctive local features (e.g., a watch) to ensure that subjects were unable to determine object identity from these cues.

Kinetic occipital region. The KO was identified in the same subjects that underwent the LOC localizer scans. We used stimuli similar to those originally used to define KO (Dupont et al., 1997). The stimuli alternated between $12 \mathrm{~s}$ epochs of transparent motion stimuli and $12 \mathrm{~s}$ epochs of kinetic boundary stimuli. Both types of stimuli were generated from random dot patterns identical to those used to localize V5/MT+. The speed and direction of local motion was the same for both stimulus types, differing only in the spatial distribution of local motion direction. In the transparent motion epochs, individual dots moved in one of two opposite directions at a speed of $5.8^{\circ} \%$, resulting in a percept of two transparent surfaces moving across one another. The axis of motion changed randomly every $0.5 \mathrm{~s}$. Four different motion axes were used $(0,45,90$, and $135^{\circ}$ from horizontal). In the kinetic boundary epochs, the stimuli were grating patterns defined by kinetic boundaries, the orientation of which changed randomly every $0.5 \mathrm{~s}$ along the same axes as the transparent motion stimuli. The grating patterns were generated by dividing the display into parallel stripes $1.5^{\circ}$ wide and displacing dots within each stripe in a single direction, parallel to the orientation of the stripe. The direction of motion alternated between adjacent stripes, giving rise to sharp motion-defined boundaries along the edges of the stripes. Both transparent motion and kinetic boundary stimuli were restricted to a circular aperture with a $10^{\circ}$ radius. Subjects underwent three scans, each consisting of 10 alternations between transparent motion epochs and kinetic boundary epochs.

\section{Results}

\section{Retinotopic organization}

Identification of retinotopic visual areas

We identified 10 distinct, retinotopically organized cortical areas, in a majority of 30 hemispheres (Figs. 1-4, supplemental Figs. $1-4$, available at www.jneurosci.org as supplemental material). Although the retinotopic maps differed from one hemisphere to another, the main features of the maps (e.g., the reversals at the vertical and horizontal meridian representations) were evident across subjects. Such intersubject differences might reflect real differences in retinotopic organization but are likely dominated by a number of methodological factors including subject scanner experience, attentional state, head motion, fixation stability, placement of image slices relative to cortical folding, cortical thickness and size relative to the functional image voxels, and so on. The retinotopic organization of eight of the identified visual areas (V1, V2, V3, hV4, VO1, V3A, V3B, and V7) has been described previously (Engel et al., 1994; Sereno et al., 1995; Tootell et al., 1995, 1997, 1998; DeYoe et al., 1996; Press et al., 2001; Brewer et al., 2005; Wandell et al., 2005). Of these areas, all except VO1 and V3B could be unambiguously identified in every subject. Here, we focus on LO1 and LO2 and their locations relative to neighboring areas $\mathrm{V} 3 \mathrm{~d}, \mathrm{~V} 3 \mathrm{~A}$, and also $\mathrm{V} 3 \mathrm{~B}$, as described by Press et al. (2001) and Wandell et al. (2005). The posterior half of LO1 corresponds to V3B of Smith et al. (1998), whereas LO2 does not correspond to any previously described visual area in humans. Because the name V3B has been used to refer to two different visual areas (see below) and because the homology with known visual areas in nonhuman primates is presently unclear, we have chosen to assign new names to both these maps. We have adopted the cortical area naming conventions proposed by Wandell et al. (2005), which has the advantage of providing anatomical information while remaining neutral with respect to function and possible cross-species homologies.

\section{Delineation and representation of visual polar angle in LO1 and $\mathrm{LO} 2$}

LO1 and LO2 were identified in 77\% (23 of 30) of the hemispheres as two adjacent mirror-image representations of the contralateral visual field located in the lateral occipital cortex anterior and lateral to $\mathrm{V} 3 \mathrm{~d}$ and posterior to $\mathrm{V} 5 / \mathrm{MT}+$ (Figs. 1-4). In the remaining $7 / 30$ hemispheres, the retinotopic organization of this region was more ambiguous. LO1 extended from the anterior boundary of V3d about halfway to area V5/MT + . Within LO1, the representation of visual polar angle progressed gradually from the lower vertical meridian toward the upper vertical meridian. In 14 of 30 hemispheres, the anterior boundary of LO1 was defined by a representation of the upper vertical meridian. In 
the other 9 of 30 hemispheres for which a polar angle map was clearly evident in LO1, the measured polar angle along the anterior LO1 boundary was closer to the horizontal than the vertical meridian. The anterior boundary of $\mathrm{LO} 1$ coincided with the posterior boundary of LO2. LO2 also contained an orderly representation of visual polar angle, extending from the upper vertical meridian (in 14 of 30 hemispheres) or the horizontal meridian (in 9 of 30 hemispheres) toward the lower vertical meridian, which defined the anterolateral boundary of LO2. LO2 did not extend into $\mathrm{V} 5 / \mathrm{MT}+$.

Location and size of $\mathrm{LO} 1$ and $\mathrm{LO} 2$

The locations of $\mathrm{LO} 1$ and $\mathrm{LO} 2$ relative to anatomical landmarks were quite variable (Fig. 4) because of the large variability in the sulcal pattern of the lateral occipital cortex (Duvernoy, 1999). The mean Talairach coordinates of LO1 or LO2 (Table 1) should thus be interpreted only as rough estimates of the true locations of these areas in individual subjects. In general, LO1 and $\mathrm{LO} 2$ were located in the fundus of the lateral (middle) occipital sulcus, with LO2 anterior to LO1. In many subjects, either or both areas also extended over the inferior and/or superior lips of the sulcus onto the lateral occipital gyrus. In those subjects where a lunate sulcus could clearly be identified, LO1 tended to be located within this sulcus. In a few hemispheres, the dorsal parts of LO1 extended into the transverse occipital sulcus, and in a small number of hemispheres, both LO1 and LO2 extended onto the inferior occipital gyrus.

LO1 and LO2 were similar in size, each subtending a cortical surface area of $\sim 300$ $\mathrm{mm}^{2}$ per hemisphere over the range of eccentricities measured (Table 2). This area was $\sim 30 \%$ of the surface area of V $1,50 \%$ of $\mathrm{V} 3$, and $75 \%$ of $\mathrm{hV} 4$ for this range of eccentricities, making $\mathrm{LO} 1$ and $\mathrm{LO} 2$ smaller than each of the previously well established retinotopic visual areas. Although the smaller sizes of LO1 and LO2 in part reflected the fact that we measured the extent of these areas for a smaller range of eccentricities than for other visual areas ( $1-4.5$ vs $\left.1-6^{\circ}\right)$, LO1 and LO2 were also considerably narrower (measured along the polar angle dimension) than other visual areas (Table 2). LO1 and LO2 were each $\sim 12 \mathrm{~mm}$ wide, corresponding to approximately half the width of $\mathrm{V} 1$.

Representation of visual field eccentricity in LO1 and LO2

The foveal representations in LO1 and LO2 were coextensive with V3d, V2d, and V1, and the periphery was represented anteriorly and dorsally (Figs. 1, 2). In about half of the hemispheres, however, the eccentricity map in LO2 showed a distinctive and unusual pattern, in that the representation of eccentricity made a sharp transition from fovea to periphery
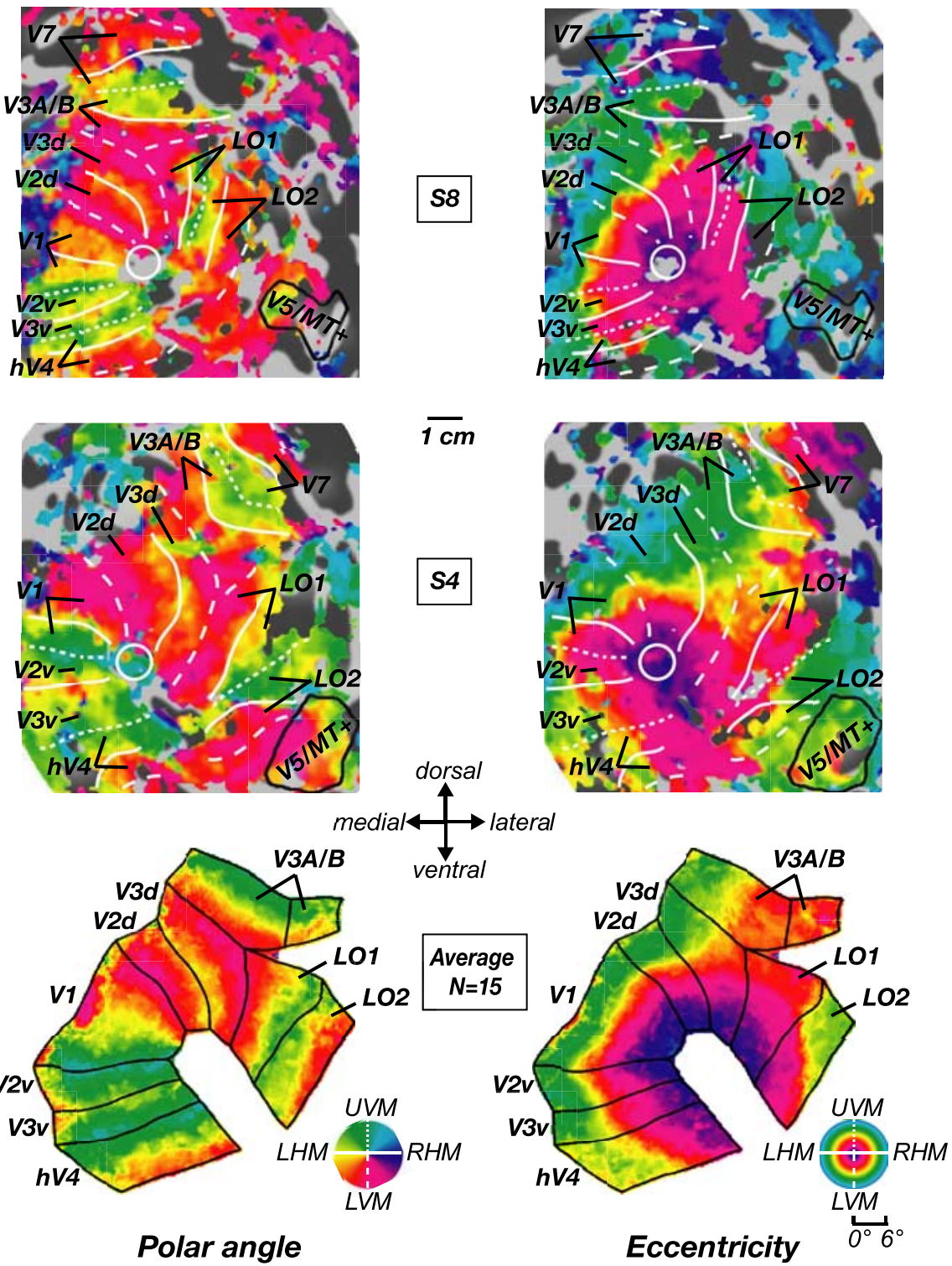

Figure 1. Right hemisphere retinotopic maps. Cortical representation of visual polar angle and eccentricity displayed on computationally unfolded and flattened patches ("flat maps") of the occipital cortex of the right hemispheres for two individual subjects (S8 and S4) and averaged across 15 hemispheres (for details of intersubject averaging, see Materials and Methods). Solid white lines, left horizontal meridian (LHM) and right horizontal meridian (RHM); dotted white lines, upper vertical meridian (UVM); dashed white lines, lower vertical meridian (LVM); white circles, approximate locations of foveal representations of V1/V2/V3. Individual subject data only shown for voxels with response coherence $>0.25$ (see Materials and Methods). In the left column, color indicates polar angle (inset legend). In the right column, color indicates eccentricity between 0 and $6^{\circ}$ (inse legend). Flat maps of polar angle in the remaining 13 right hemispheres are available as online supplemental information (supplemental Figs. 1, 2, available at www.jneurosci.org as supplemental material).

(Figs. 1, 2, supplemental Figs. 2, 4, available at www.jneurosci. org as supplemental material). This finding replicated previous observations by Tootell and Hadjikhani (2001), who subdivided the cortex between $\mathrm{V} 3 \mathrm{~d}$ and $\mathrm{V} 5 / \mathrm{MT}+$ along this transition boundary into two regions, LOC and LOP, representing central and peripheral eccentricities, respectively. LOC and LOP likely correspond to the foveal and peripheral representations of LO2 and LO1.

Skewed representations of the visual field in $\mathrm{LO} 1$ and $\mathrm{LO} 2$ Unlike earlier visual areas, but similar to V7 (Tyler et al., 2005b), the polar angle and radial components of the maps in LO1 and LO2 were not orthogonal to one another. Isoeccentricity contours in both areas ran at an acute angle to isoangle contours, 


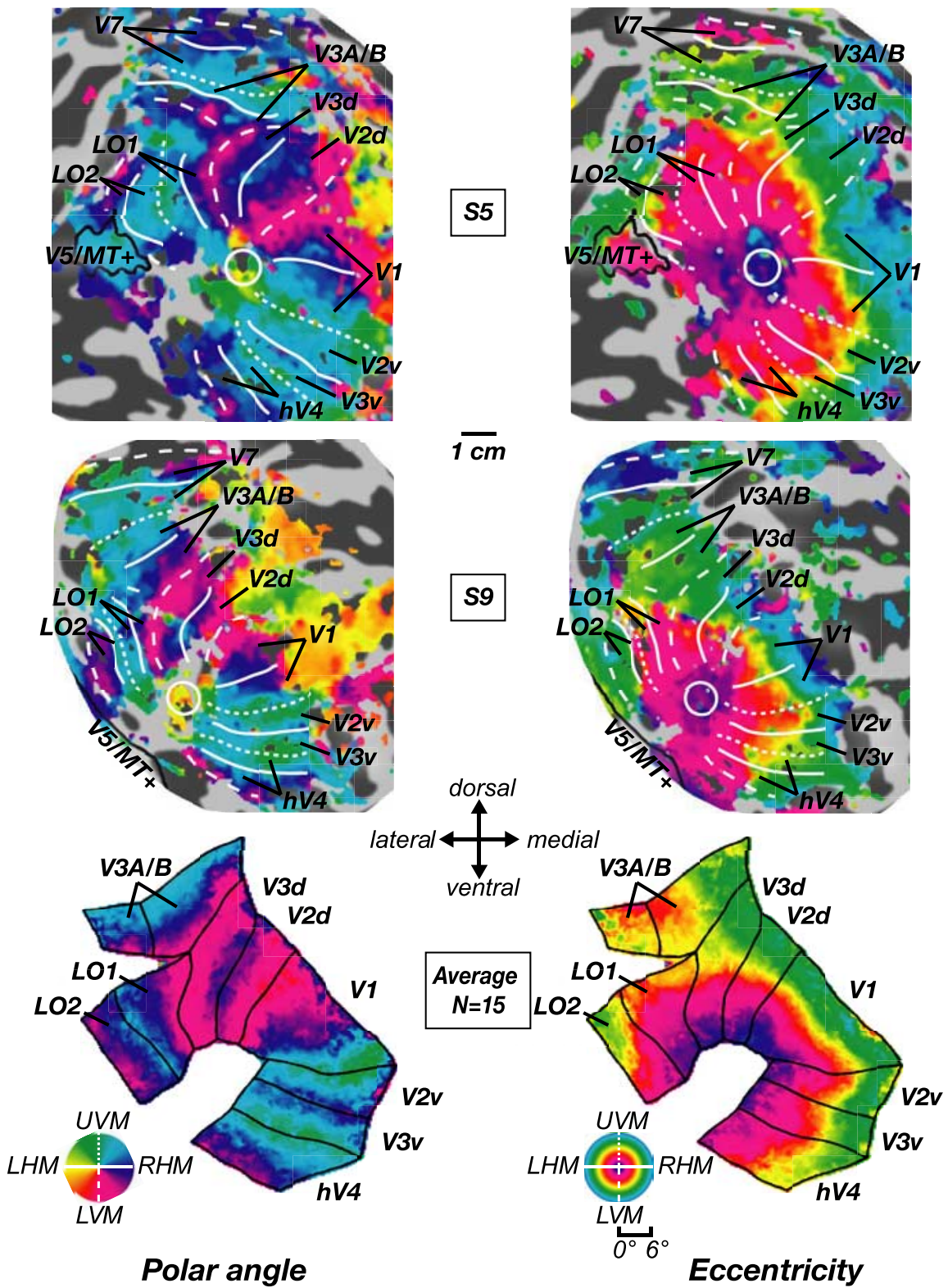

Figure 2. Left hemisphere retinotopic maps of subjects $\$ 5$ and $\$ 9$, and averaged across 15 hemispheres. Conventions, abbreviations, and legend are as in Figure 1. Flat maps of polar angle in the remaining 13 right hemispheres are available as online supplemental information (supplemental Figs. 3, 4, available at www.jneurosci.org as supplemental material).

denly from fovea to periphery when sampled at the relatively coarse resolution of current fMRI methods.

\section{Visual field topography of neighboring} visual areas

The lower vertical meridian representation at the anterior border of V3d had a characteristic $\mathrm{Y}$ shape that defined the boundaries between four visual areas: V3d, V3A, V3B, and LO1 (Figs. 1-3). About midway along the length of the V3d boundary, the meridian representation bifurcated into one branch extending dorsally and posteriorly along V3d, and another branch extending anteriorly away from V3d. This pattern was observed in almost every subject, although the diverging branch was less distinct in some hemispheres (Figs. 1, subject S4, 2, subject S9). A similar bifurcation of the vertical meridian representation in $\mathrm{V} 3 \mathrm{~d}$ has been reported in the macaque (Gattass et al., 1988; Fize et al., 2003). V3A and V3B each contained a full map of the contralateral hemifield such that their dorsal/anterior boundary (with V7) represented the upper vertical meridian. Consistent with previous reports (Press et al., 2001; Wandell et al., 2005), the boundary between V3A and V3B was defined by their shared foveal representation, separate from the central foveal representation of $\mathrm{V} 1, \mathrm{~V} 2$ and $\mathrm{V} 3$, with the radial components of the two maps extending in opposite directions (medially in V3A and laterally in V3B) (Figs. 1-3). The clarity of the foveal representation in V3A/B varied considerably between subjects, from crisp and well defined in some subjects and hemispheres (supplemental Figs. 2, right hemispheres of subjects S5, S6 S10, S12, S13; 4, left hemispheres of subjects S1, S2, S6, S7, S10, S13, S14, S15, available at www.jneurosci. org as supplemental material) to very faint or nonexistent in others. This variability may have been caused by the limited eccentricity range of our stimuli, as previous studies of $\mathrm{V} 3 \mathrm{~A} / \mathrm{B}$ were performed using larger stimuli $\left(>15^{\circ}\right)$ (Press et al., 2001).

resulting in a representation of the visual field in both areas that was noticeably skewed, particularly in LO2 (Figs. 1, 2, 8). Although the eccentricity and polar angle maps in LO2 appeared nearly parallel in some subjects, the mean angle between the maps in this area, measured in the nine subjects ( 18 hemispheres) with the clearest polar angle maps in LO1 and LO2, differed significantly from zero [mean angle $15.3^{\circ} ; p<0.05$, nonparametric test of angular differences (Fisher, 1993)]. A similarly distorted visual field representation has been reported in dorsal V4 of the macaque (Gattass et al., 1988; Fize et al., 2003) whose location relative to V3d and MT parallels that of LO1/LO2. The pronounced skew of the visual field map in LO2 could, in principle, account for the apparent sudden transition between central and peripheral eccentricity representations in $\mathrm{LO} 2$; given the small size of LO2 (Table 2), such a skewed map could appear to jump sud-
For those subjects for whom we could unambiguously identify the boundary between V3A and V3B, V3B was always dorsal and anterior to the vertical meridian bifurcation. Because we could not confidently identify the boundary between V3A and V3B in all subjects, we used a single region of interest encompassing both of these areas, which we refer to as $\mathrm{V} 3 \mathrm{~A} / \mathrm{B}$, in the analyses of stimulus selectivity described below.

The posterior half (i.e., the lower quadrant representation) of $\mathrm{LO} 1$ corresponds to a region that was originally defined as V3B by Smith et al. (1998). We have nonetheless chosen to assign a new name to this area, for the following reasons. First, as described in detail in the following sections, our data suggest that LO1 contains a full hemifield representation, not just a map of the lower quadrant as originally suggested for V3B (Smith et al. 1998). Also, 
the original report of $\mathrm{V} 3 \mathrm{~B}$ only described the representation of polar angle, but not of eccentricity in this area, making exact comparison of our data with those of Smith et al. (1998) difficult. Second, more recent studies (Press et al., 2001; Wandell et al., 2005) have used the label V3B to describe a different area that shares a foveal representation with $\mathrm{V} 3 \mathrm{~A}$, in a location that is dorsolateral to LO1 and LO2. Their use of the label reflects a different interpretation of the results of Smith et al. (1998). In the original report by Smith et al. (1998), V3B and V3A were described as separated by a gap in the polar angle map anterior to V3d. Press et al. (2001) interpreted this "silent" zone as corresponding to a foveal representation common to V3A and V3B. This was further elaborated by Wandell et al. (2005), who suggested that $\mathrm{V} 3 \mathrm{~A}$ and $\mathrm{V} 3 \mathrm{~B}$ form a visual field map cluster with eccentricity representations extending in opposite directions from a common fovea dorsolateral to, and separate from, the common foveal representation of V1/V2/V3. However, although the figures of Smith et al. (1998) do not show the eccentricity representation in $\mathrm{V} 3 \mathrm{~B}$, the foveal representation in $\mathrm{V} 3 \mathrm{~B}$ as originally defined by Smith et al. (1998) was coextensive with that in V3d (Andy Smith, personal communication). The key feature distinguishing these two definitions of $\mathrm{V} 3 \mathrm{~B}$ is the bifurcating vertical meridian representation anterior to V3d, a feature that we consistently observed in our results. Whereas both definitions agree that the posterior boundary of $\mathrm{V} 3 \mathrm{~B}$ aligns with this vertical meridian representation, they locate V3B on the opposite side of the bifurcation, ventral (Smith et al., 1998) or dorsal (Tyler et al., 2005b; Wandell et al., 2005). Consistent with our observations, Tyler et al. (2005b) argued that the area defined as V3B by Press et al. (2001) extends along this bifurcating meridian away from $\mathrm{V} 3 \mathrm{~d}$ and does not extend into the cortical region between $\mathrm{V} 3 \mathrm{~d}$ and $\mathrm{V} 5 / \mathrm{MT}+$ where Smith et al. (1998) had originally located V3B. The two definitions of V3B thus refer to two different cortical areas. We were able to identify the visual field map referred to as V3B by Wandell et al. (2005) in several subjects and chose to adopt their naming convention. Note that the area we refer to as LO1 has a different visual field representation than area V3B as defined by Smith et al. (1998) and, thus, is not identical to (although it partially overlaps with) this area. The choice of terminology is therefore mainly a matter of convention and does not affect the interpretation of our data.

In some hemispheres, LO2 directly abutted V5/MT+, whereas the two areas appeared to be separated by a short distance in other hemispheres. Because the boundaries of V5/MT+ were defined by thresholding a statistical map (using the same threshold for all subjects), it is possible that this variability merely reflected intersubject differences in the strength of the evoked $\mathrm{fMRI}$ responses in $\mathrm{V} 5 / \mathrm{MT}+$. Following previous reports (Huk et al., 2002), a retinotopically organized subregion of V5/MT + was evident in some hemispheres, which is believed to be the human homolog of macaque area MT. In those hemispheres in which the polar angle map in MT was clearly distinct (right hemisphere of
S3, S4, S6, left hemisphere of S11), the anterior boundary of LO2 was coextensive with the posterior (lower vertical meridian) boundary of MT.

\section{Atlas of visual field topography}

Using the parameters of the atlas fit, the polar angle and eccentricity maps of individual subjects were aligned and averaged across subjects (Figs. 1, 2, bottom rows). Visual area boundaries are clearly visible in these average maps as reversals (local minima or maxima) in the gradient of the polar angle representation. The goodness of the atlas fit is evident in the close alignment of these reversals with the atlas boundaries (black lines). The primary purpose of the atlas fitting procedure was to align data across subjects and reduce the subjective bias of manually identifying boundaries in the flat maps. Given the nature of the fitting algorithm, it is to be expected that the average fit should resemble the model. The atlas fits are, thus, not direct evidence of the validity of this model, and we base our conclusions about the existence of LO1 and LO2 not on the atlas fits, but on the raw data. However, the atlas fits nonetheless helped validate key properties of the model, including the orderly retinotopic organization in $\mathrm{LO} 1$ and $\mathrm{LO} 2$. First, the atlas fits for LO1 and LO2 were qualitatively similar to those in other visual areas, which are known to be retinotopically organized (i.e., the maps of LO1 and LO2 in the averaged data were smooth and continuous, and the radial and polar dimensions, although not orthogonal, were nonparallel to one another). Second, the root mean square error (RMSE) between the fitted atlas templates and the data in LO1 and LO2 were on par with that in other visual areas (supplemental Fig. 6, available at www.jneurosci.org as supplemental material). Third, the fit- 
$\boldsymbol{A}$

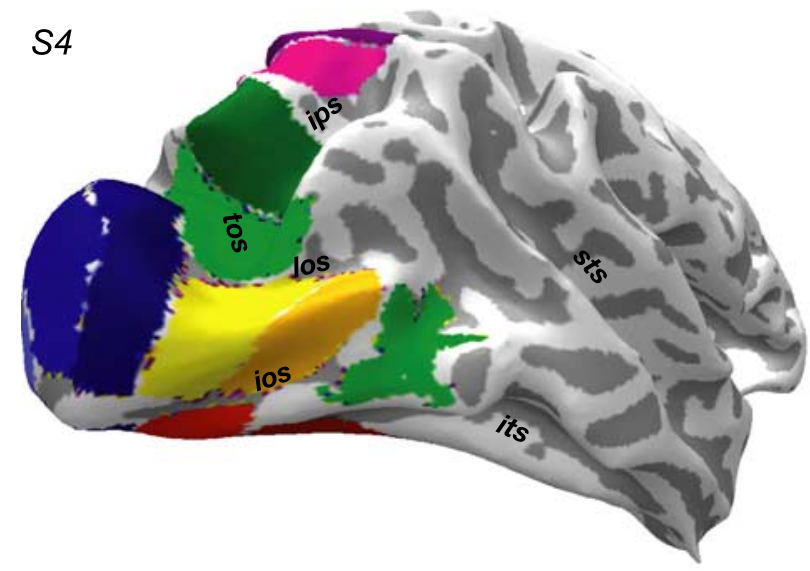

B
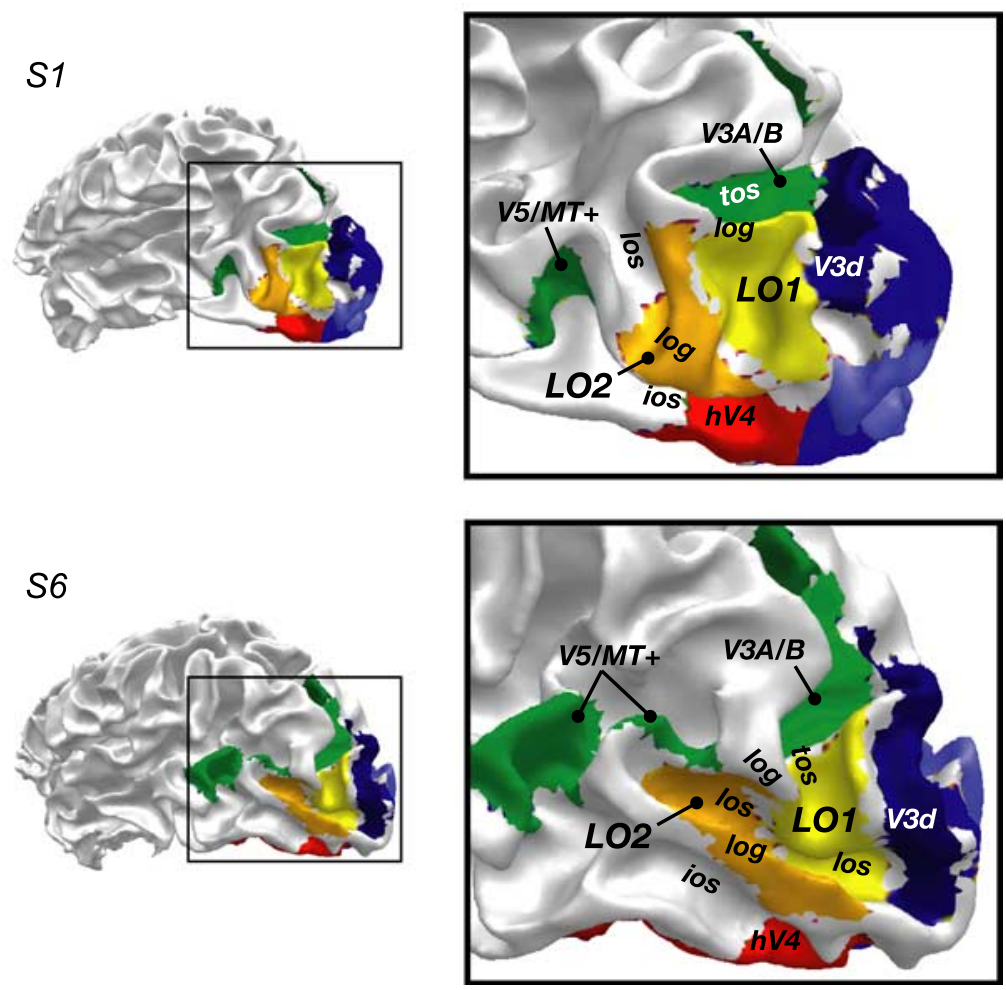

Figure 4. Anatomical locations of L01 and L02. A, Locations of L01 and L02 and neighboring visual areas displayed on a partially inflated brain of a representative subject. los, lateral occipital sulcus; log, lateral occipital gyrus; tos, transverse occipital sulcus; ios, inferior occipital sulcus; its, inferior temporal sulcus; sts, superior temporal sulcus; ips, intraparietal sulcus. $\boldsymbol{B}$, Variability in the locations of L01 and L02 across subjects relative to local anatomical landmarks. For subject S1, L01 and L02 are located mostly on the crest of the lateral occipital gyrus/gyri; for subject S6, L01 and L02 are located mostly on the fundus of the lateral and transverse occipital sulcus/sulci.

ting procedure was prevented from overfitting by simultaneously having to fit polar angle and eccentricity templates to the data, with additional elasticity constraints. Fourth, the atlas fitting procedure yielded a result that was not specified in the atlas, but which was consistent with previous studies of visual topography (Wandell et al., 2005): the average eccentricity maps of V3A/B contained a foveal confluence (particularly evident in Fig. 2, bottom row, but also visible in Fig. 1), even though the fitted atlas did not include eccentricity templates for $\mathrm{V} 3 \mathrm{~A} / \mathrm{B}$.

Cortical magnification

Within the range of eccentricities spanned by our stimuli, cortical magnification functions in the eccentricity dimension in LO1

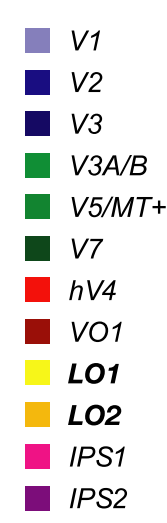

and LO2 were similar to those in lower-tier visual areas (Fig. 5). In all visual areas examined, we found a marked overrepresentation of the fovea. The measurements in V1-V3 were in general agreement with the consensus of previous reports (Horton and Hoyt, 1991; Sereno et al., 1995; Engel et al., 1997; Dougherty et al., 1999; Duncan and Boynton, 2003), and our numerical estimates of cortical magnification factors in these areas agreed with the consensus opinion in the literature (Horton and Hoyt, 1991; Engel et al., 1997). Our data also suggested that the representation of visual space was much more compressed along the polar angle axis than along the eccentricity axis in all areas. For instance, in V1, eccentricities between 1 and $5^{\circ}$ were represented within $\sim 30 \mathrm{~mm}$ of the cortex, or $\sim 7 \mathrm{~mm} / \mathrm{deg}$ (Fig. 5). The average width of $\mathrm{V} 1$ along the polar angle dimension, measured over a slightly larger range of eccentricities $\left(1-6^{\circ}\right)$, was $\sim 25 \mathrm{~mm}$ (Table 2 ). At an average eccentricity of $3.5^{\circ}$, this corresponds to a visual field distance of $3.5 \pi=11^{\circ}$ visual angle, or $\sim 2.3 \mathrm{~mm} / \mathrm{deg}$, indicating that on average, the representation of the visual field in V1 was three times more compressed along the polar angle axis than along the eccentricity axis. For higher-tier areas, the relative compression was even larger (e.g., in LO1, the corresponding numbers were $1.5 \mathrm{~mm} / \mathrm{deg}$ along the polar angle axis vs $10 \mathrm{~mm} / \mathrm{deg}$ along the eccentricity axis, assuming a hemifield representation in this area). Different cortical magnification along the polar angle and eccentricity axes have been reported previously in V1 of macaques (Van Essen et al., 1984; Adams and Horton, 2003) and ferrets (Yu et al., 2005).

\section{Response field size}

Consistent with previous estimates of response field size in human visual areas (Smith et al., 2001) and single-unit measurements of receptive field sizes in macaque visual areas, we found that voxel response field sizes increased with visual field eccentricity (Fig. 6A). The rate of increase (Fig. $6 A$, slopes of best-fit lines) was greater in higher-tier visual areas, such as LO1 and LO2, than in early visual areas (Fig. 6B). Note that response field sizes and the rate of increase of response field sizes with eccentricity were three to four times larger in the polar dimension than in the radial dimension (Fig. 6B). This was because of the more compressed visual field representation along the polar angle axis than along the eccentricity (radial) axis (see above). Note also that the response field sizes increased more rapidly at low eccentricities where the cortical magnification was the highest (Fig. 6A).

Visual field coverage in $\mathrm{LO} 1$ and $\mathrm{LO} 2$

In almost half (14 of 30) of the hemispheres examined, both LO1 and LO2 spanned nearly the full range of contralateral polar 
Table 1. Talairach coordinates of the centroids of L01 and L02 (mean \pm SD and range across all 15 subjects), based on boundaries defined by atlas fitting procedure (see Materials and Methods)

\begin{tabular}{|c|c|c|c|c|c|c|}
\hline & \multicolumn{3}{|c|}{ Left hemisphere } & \multicolumn{3}{|c|}{ Right hemisphere } \\
\hline & $x$ & $y$ & $z$ & $x$ & $y$ & $z$ \\
\hline \multicolumn{7}{|l|}{ L01 } \\
\hline $\begin{array}{l}\text { Mean } \\
\text { Range }\end{array}$ & $\begin{array}{l}-31 \pm 3.9 \\
(-37,-26)\end{array}$ & $\begin{array}{l}-90 \pm 5.2 \\
(-101,-82)\end{array}$ & $\begin{array}{l}1.4 \pm 6.9 \\
(-12,11)\end{array}$ & $\begin{array}{l}32 \pm 4.2 \\
(24,38)\end{array}$ & $\begin{array}{l}-89 \pm 5.1 \\
(-98,-81)\end{array}$ & $\begin{array}{l}2.6 \pm 6.5 \\
(-8,13)\end{array}$ \\
\hline \multicolumn{7}{|l|}{ L02 } \\
\hline $\begin{array}{l}\text { Mean } \\
\text { Range }\end{array}$ & $\begin{array}{l}-38 \pm 3.4 \\
(-43,-31)\end{array}$ & $\begin{array}{l}-83 \pm 6.4 \\
(-92,-67)\end{array}$ & $\begin{array}{l}-0.1 \pm 7.1 \\
(-13,11)\end{array}$ & $\begin{array}{l}38 \pm 4.4 \\
(32,46)\end{array}$ & $\begin{array}{l}-82 \pm 5.1 \\
(-89,-72)\end{array}$ & $\begin{array}{l}0.6 \pm 7.2 \\
(-13,12)\end{array}$ \\
\hline
\end{tabular}

Table 2. Surface area (millimeters squared) and width (millimeters) of retinotopic visual areas representing $1-6^{\circ}$ eccentricity (for L01 and L02, 1-4.5 eccentricity), mean, and SD across all 15 subjects

\begin{tabular}{|c|c|c|c|c|c|c|c|c|}
\hline & Hemisphere & V1 & V2 & V3 & $\mathrm{V} 3 \mathrm{~A} / \mathrm{B}$ & hV4 & L01 & L02 \\
\hline \multirow[t]{7}{*}{ Surface area } & Left & 1162 & 1053 & 937 & 592 & 482 & 321 & 289 \\
\hline & SD & 341 & 267 & 201 & 129 & 122 & 147 & 76 \\
\hline & Right & 1161 & 1050 & 1010 & 606 & 515 & 340 & 374 \\
\hline & SD & 346 & 261 & 226 & 116 & 99 & 129 & 112 \\
\hline & Both & 2323 & 2102 & 1946 & 1197 & 997 & 662 & 663 \\
\hline & SD & 647 & 492 & 365 & 181 & 166 & 230 & 161 \\
\hline & $\%$ of V1 & 100 & 90 & 84 & 52 & 43 & 28 & 29 \\
\hline \multirow[t]{7}{*}{ Width } & Left & 25.0 & 23.3 & 22.1 & 13.1 & 16.3 & 12.8 & 11.4 \\
\hline & SD & 5.1 & 3.3 & 3.7 & 2.2 & 4.5 & 3.7 & 2.6 \\
\hline & Right & 24.2 & 22.5 & 22.8 & 13.6 & 14.8 & 12.7 & 13.1 \\
\hline & SD & 4.1 & 3.0 & 2.7 & 2.1 & 3.3 & 3.8 & 2.5 \\
\hline & Both & 24.6 & 22.9 & 22.5 & 13.4 & 15.5 & 12.7 & 12.2 \\
\hline & SD & 4.6 & 3.1 & 3.2 & 2.1 & 4.0 & 3.7 & 2.6 \\
\hline & $\%$ of V1 & 100 & 93 & 91 & 54 & 63 & 52 & 50 \\
\hline
\end{tabular}

Area boundaries are defined by atlas fitting procedure (see Materials and Methods). Width along polar angle axis is defined as average distance along isoeccentricity contours between area boundaries corresponding to visual field meridians.

angles, suggesting that each of these areas contained a complete map of the contralateral hemifield (Figs. 1, 2, supplemental Figs. 1,3 , available at www.jneurosci.org as supplemental material). In 9 of 30 hemispheres, the range of polar angles in either or both of LO1 and LO2 was less than a full hemifield and closer to a quadrant. There are two possible explanations for this result. One is that the variability in the measured visual field coverage reflects a genuine underlying intersubject variability in the extent of visual field representation in these areas. Another possibility is that methodological limitations and measurement errors caused a constant visual field representation to appear variable across subjects. Given that there is little evidence for large intersubject variability in the extent of visual field coverage in earlier visual areas (e.g., V1-V3), and that our measurements of visual field representation are necessarily indirect, we think it more likely that the variability is caused by methodological limitations, as detailed below. The relevant question then becomes, what is the extent of the underlying visual field representation in LO1 and LO2? To answer this issue we characterized the visual field coverage of these areas in two ways, one of which estimated the full extent of response field coverage for each visual area (Fig. 7) and the other of which characterized the distribution of response field centers (Fig. 8).

First, based on the estimated voxel response field sizes, we calculated the total visual field coverage of each visual area (Fig. 7). As is apparent in this figure, both the upper and lower quadrants of the contralateral hemifield were represented in LO1 and $\mathrm{LO} 2$. The visual representations in both LO1 and LO2 were similar to that in V1, suggesting that LO1 and LO2 each contained a representation of the contralateral hemifield. By comparison, the visual field representations in V3d and V3v calculated by this method were restricted to the corresponding (lower and upper) quadrants. For both V3d and V3v, the visual field representation extended only marginally across the horizontal meridian, with little or no overlap between the two representations. Furthermore, the visual field coverage in LO1 and LO2 was more extensive than that of hV4, an area which is believed to represent a full hemifield (Brewer et al., 2005).

Second, we used the atlas fitting procedure to compare two models of visual field topography in LO1 and LO2 (one assuming full hemifield representations, and the other assuming quadrant representations), and found that the full hemifield model was a better fit to the data than the quadrant model (supplemental Fig. 6 , available at www.jneurosci.org as supplemental material). The goodness of fit was assessed by measuring the RMSE between model and data, for each visual area and each model. The RMSE for LO1 and LO2 was similar to other visual areas, demonstrating that the atlas provided an equally good characterization of the topography in LO1 and LO2 as in other well established visual areas. For LO2, the RMSE was significantly lower for the hemifield than for the quadrant model (paired $t$ test, $\mathrm{df}=29, p<$ $0.01)$. For LO1 the difference was smaller and showed only a nonsignificant trend (paired $t$ test, $\mathrm{df}=29, p=0.14$ ).

The atlas fits were visualized in LO1 and LO2, and (for comparison) in $\mathrm{V} 1, \mathrm{~V} 2, \mathrm{~V} 3$, and $\mathrm{hV} 4$, by averaging the polar angle and eccentricity maps across all hemispheres and subjects (Fig. 8). Specifically, we averaged the individual maps of polar angle and eccentricity in the canonical (template) coordinate system of the atlas (see Materials and Methods), and then plotted polar angle along the atlas isoeccentricity axis, collapsing the data across eccentricity (Fig. 8C). The representations in $\mathrm{LO} 1$ and LO2 exhibited a range of polar angles that was less than a full hemifield [i.e., 


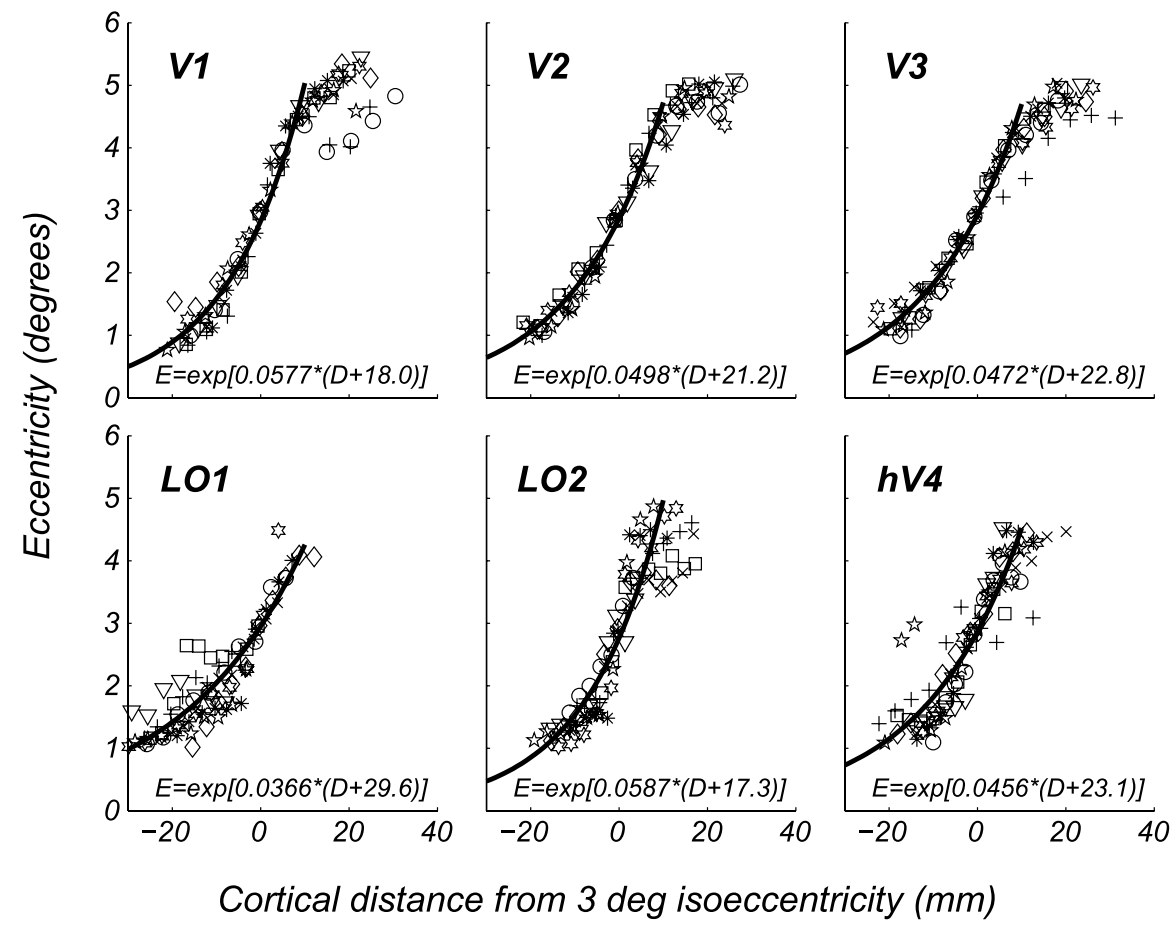

Figure 5. Cortical magnification functions for V1, V2, V3, L01, L02, and hV4. Plot symbols, eccentricity, and cortical distance measurements from nine subjects (different plot symbols indicate different subjects) are shown. Solid curves and equations show parametric fits to the data pooled across subjects, where $E$ is eccentricity in degrees, $D$ is cortical distance in millimeters, and $a$ and $b$ are constants. Cortical distances have been aligned to an origin corresponding to the $3^{\circ}$ isoeccentricity representation (see Materials and Methods). Because of the reduced stimulus duty cycle close to the edge of the stimulus aperture (beyond $5^{\circ}$ from the center of gaze), there was a systematic underestimation of eccentricity at more peripheral locations (evidenced by an apparent change in the slope of eccentricity versus distance beyond $\sim 5^{\circ}$ or $10 \mathrm{~mm}$ from the origin). Data from these peripheral locations were not included in the fits (solid lines).

the mean polar angle profile (Fig. $8 C$, red solid curve) exhibited a range of less than $\pi$ radians], but more than a quadrant (i.e., more than $\pi / 2$ radians), including parts of both the upper and lower quadrants (Figs. 7, 8C). The range of polar angles in LO1 and LO2 was approximately twice that of neighboring area V3d, which represents only the lower quadrant of the visual field (Fig. 8C).

Although the range of polar angles in LO1 and LO2 was smaller than a hemifield, we argue that this was to be expected given that the measured response phase at each voxel corresponded to the center (not the full extent) of the voxel's response field. In the macaque monkey, the receptive fields of neurons along the vertical meridian boundaries of V2, V3, and V4 extend only $3-4^{\circ}$ into the ipsilateral hemifield (Gattass et al., 1981, 1988). Where receptive field sizes are larger than this dimension (e.g., at higher eccentricities and in higher-tier areas), the vertical meridian is represented by neurons with receptive field centers displaced away from the meridian. If the same were true for humans, such that neuronal receptive fields along the boundaries of LO1 and LO2 do not extend symmetrically into the ipsilateral hemifield, the polar angle representation along these boundaries would be displaced toward the horizontal meridian. Hence, even if it were possible to record the pointwise polar angle representation in human visual cortex without the inevitable spatial blurring introduced by sampling and hemodynamics (Engel et al., 1997), one would expect the measured range of polar angles in LO1 and LO2 to be less than a full hemifield.

Moreover, the range of polar angles will be further reduced by spatial blurring inherent in the fMRI measurements. Neurons representing the vertical meridian (i.e., near a reversal in polar angle such as that between LO1 and LO2), are surrounded on either side by neurons with receptive fields off the vertical meridian in the same hemifield. Averaging the responses of neighboring neurons along such a boundary will yield a mean response phase (and consequently mean polar angle) that is shifted toward the horizontal meridian, by an amount that is roughly proportional to the amount of spatial averaging. The shift will be greater for small or narrow areas in which polar angle varies more rapidly over a given distance. Consistent with this prediction, LO1 and LO2, which showed the greatest shift in mean polar angle, were substantially smaller in size than other visual areas (Table 2). Furthermore, the measured range of polar angles in each area was proportional to the surface area of LO1 and LO2 in individual hemispheres, suggesting that the reduced range of polar angles in these areas could at least in part be explained by their small size (supplemental Fig. 7, available at www.jneurosci.org as supplemental material). The bias evident in the mean polar angle profile (Fig. 8C) was no doubt also exacerbated by averaging across all 30 hemispheres; any misalignment in the atlas fits would have shifted the mean asymmetrically toward the horizontal meridian. In addition, the data would have been further blurred by subject movement, misregistration between functional data and anatomical data for each subject, and misregistration between subjects. Finally, receptive field scatter, which is greater in higher tier visual areas, would act like additional spatial smoothing. Note that these blurring effects can only result in a reduction, not an expansion, of the range of polar angle representation in an area. If the boundary between LO1 and LO2 had been defined by the horizontal meridian (implying a quadrant representation in each of these areas), the effect of spatial and receptive field scatter-induced blurring would have predicted a shift of the mean measured polar angle along this boundary to a value intermediate between the horizontal meridian and the lower vertical meridian (i.e., a range smaller than a quadrant). Instead, the mean polar angle along this boundary had a value intermediate between the horizontal meridian and the upper vertical meridian (i.e., a range larger than a quadrant). This result is consistent with spatial and/or receptive field scatter-induced blurring of a full hemifield map, but not a quadrant map, in LO1 and LO2.

\section{Stimulus selectivity}

LO1 and LO2 are not motion selective

By comparing the responses to moving and stationary dot patterns, we identified three motion-preferring cortical regions: one in $\mathrm{V} 1 / \mathrm{V} 2$, one in $\mathrm{V} 3 / \mathrm{V} 3 \mathrm{~A} / \mathrm{B}$, and a third region, corresponding to $\mathrm{V} 5 / \mathrm{MT}+$ (Fig. 9A). Neither LO1 nor LO2 responded strongly to motion, despite the fact that they were flanked by V3, V3A, V3B, and $\mathrm{V} 5 / \mathrm{MT}+$. The mean responses averaged across subjects to moving dot patterns in LO1 and LO2 were not significantly different from the responses to stationary dots (Fig. 9D) (two-tailed 
$\boldsymbol{A}$

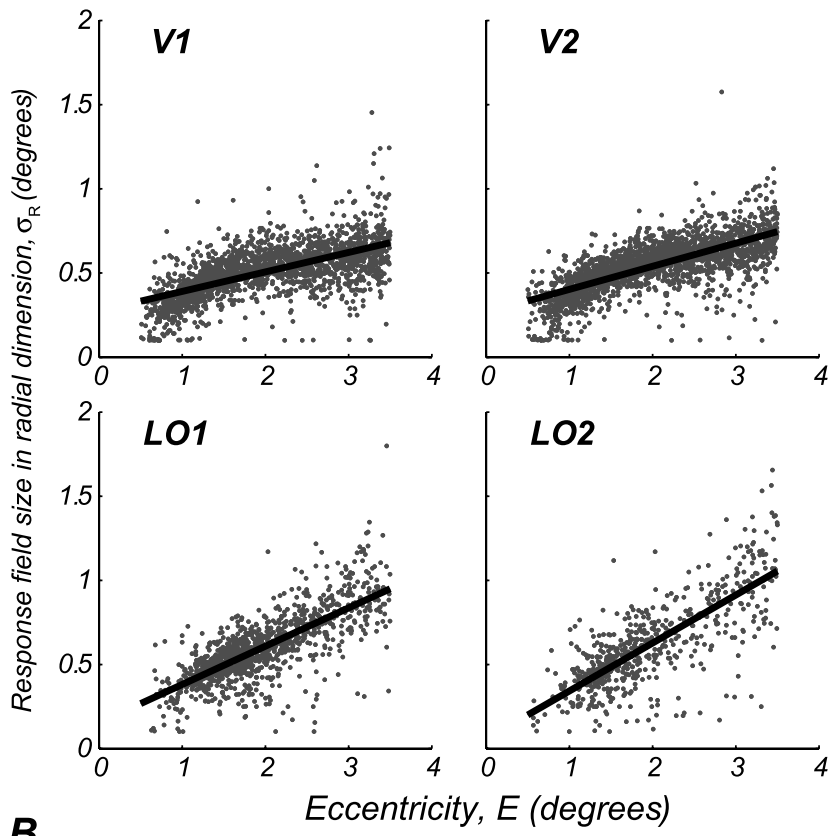

B

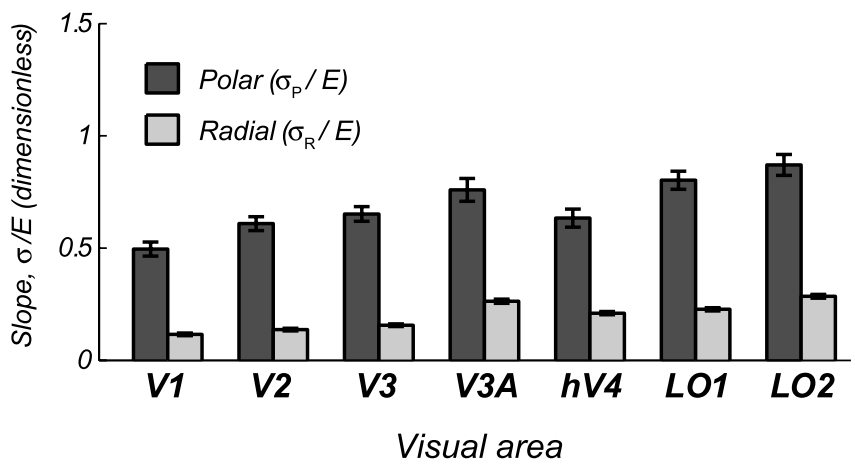

Figure 6. L01 and L02 have large voxel response fields. $\boldsymbol{A}$, Voxel response field size along radial (eccentricity) dimension ( $\sigma_{R}$, SD of Gaussian fits to expanding/contracting ring data) as a function of eccentricity in areas V1, V2, L01, and L02. Response field sizes were estimated for individual voxels in 28 hemispheres with response fields centered between 0.5 and $3.5^{\circ}$ eccentricity, and with response coherence $>0.25$ (see Materials and Methods). Lines are least squares fits. $\boldsymbol{B}$, Slopes of best-fit lines for each visual area. The light bars show the radial component of response field size $\sigma_{R}$, based on least squares fits such as those shown in $A$. The dark bars show the polar angle component of response field size $\sigma_{\mathrm{p}}$. Error bars indicate SEM across subjects and hemispheres. Response field sizes increase more rapidly with eccentricity (steeper slopes) in higher-tier visual areas.

$t$ test, $\mathrm{df}=6, p=0.62$ and $p=0.06$ for $\mathrm{LO} 1$ and $\mathrm{LO} 2$, respectively), implying that neither area was responsive to visual motion.

\section{Both LO1 and LO2 respond to motion boundaries}

To determine whether LO1 or LO2 overlapped with the kinetic occipital region, we measured the fMRI responses to motion (or kinetic) boundary stimuli relative to a transparent motion control using a protocol and stimuli similar to that used in the original description of KO (Dupont et al., 1997). Consistent with previous studies, we found that motion boundaries evoked the strongest responses in cortex anterior to V3 (Fig. 9B). Both LO1 and $\mathrm{LO} 2$ responded significantly more strongly to motion boundaries than to transparent motion (two-tailed $t$ test, $\mathrm{df}=6$, $p<0.001$ and $p<0.001$ for LO1 and LO2, respectively). The region of evoked activity extended across multiple visual areas, including $\mathrm{LO} 1, \mathrm{LO} 2, \mathrm{~V} 3 \mathrm{~A} / \mathrm{B}, \mathrm{V} 7$, parts of $\mathrm{hV} 4$ and $\mathrm{VO} 1$, and also extended into cortical regions anterior to known retinotopic areas (Fig. 9B). The magnitude of responses to motion boundaries differed significantly across visual areas (one-way ANOVA, $\left.F_{(9,60)}=8.32, p \approx 0\right)$, with the strongest responses in LO1, LO2, $\mathrm{V} 3 \mathrm{~A} / \mathrm{B}$, and V3 (multiple comparison of means, $p<0.05$ ). LO1 and $\mathrm{LO} 2$ were, thus, part of $\mathrm{KO}$, although our results also show that $\mathrm{KO}$ is not a single region but includes several retinotopic visual areas.

\section{LO1 and LO2 are part of the LOC}

We tested whether LO1 or LO2 were part of the LOC, defined operationally as the cortical regions showing stronger fMRI responses to images of common objects or faces, compared with scrambled images of the same stimuli. A large region of visual cortex, including LO1 and LO2, showed a stronger response to intact than to scrambled images of objects (Fig. 9C). Most of these regions were anterior to retinotopically defined areas, but also included other higher-tier retinotopic extrastriate areas, such as $\mathrm{hV} 4, \mathrm{~V} 3 \mathrm{~A}$, and VO1. Object-selective responses in LO1 and LO2 were highly significant (two-tailed $t$ test, $\mathrm{df}=6, p<0.001$ and $p<0.001$, respectively). The magnitude of responses to intact images differed significantly across visual areas (one-way ANOVA, $F_{(9,60)}=46.4, p \approx 0$ ), and was higher in LO2 than in any other retinotopic area, including LO1 (multiple comparison of means, $p<0.05$ ). We also compared responses to objects with responses to faces, a contrast known to divide the LOC into objectpreferring posterior/dorsal regions and face-preferring anterior/ ventral regions (Grill-Spector et al., 1999, 2001; Hasson et al., 2003; Tsao et al., 2003). Both LO1 and LO2 responded significantly more strongly to images of objects than to images of faces (two-tailed $t$ test, $\mathrm{df}=6, p<0.001$ and $p<0.005$ for LO1 and LO2, respectively). These results confirmed that LO1 and LO2 were part of the LOC, with object-selective response properties consistent with those found in previous studies of the posterior LOC.

\section{Orientation-selective adaptation in LO1, but not LO2}

In a separate study, details of which have been reported previously (Larsson et al., 2006), we measured orientation-selective adaptation to sinusoidal gratings defined by luminance, contrast, or orientation cues. The results showed a marked dissociation between the response properties of LO1 and LO2. Whereas LO1 showed robust orientation-selective adaptation to all three stimulus types, LO2 did not exhibit orientation selectivity to any of the stimuli.

\section{Discussion}

Retinotopic organization of lateral occipital cortex

We found that the human lateral occipital cortex between dorsal $\mathrm{V} 3$ and V5/MT + contains two well organized maps of the contralateral visual hemifield. Based on retinotopy and unique functional properties, we propose that these maps constitute two visual areas, which we have named LO1 and LO2.

Our results clearly demonstrate that the human lateral occipital cortex between V3d and V5/MT + is retinotopically organized, with orderly representations of both polar angle and eccentricity. This region has been characterized previously as essentially nonretinotopic (Grill-Spector et al., 1998b; Tyler et al., 2005a), as containing only a coarse eccentricity bias with no clear map of polar angle (Levy et al., 2001; Tootell and Hadjikhani, 2001), or as containing a coarse retinotopic map with an overemphasized representation of the lower vertical meridian with little or no representation of the upper vertical meridian (Tyler et al., 2005b). One previous study has reported the existence of an orderly polar angle map, area $\mathrm{V} 3 \mathrm{~B}$, in this region. $\mathrm{V} 3 \mathrm{~B}$ was 

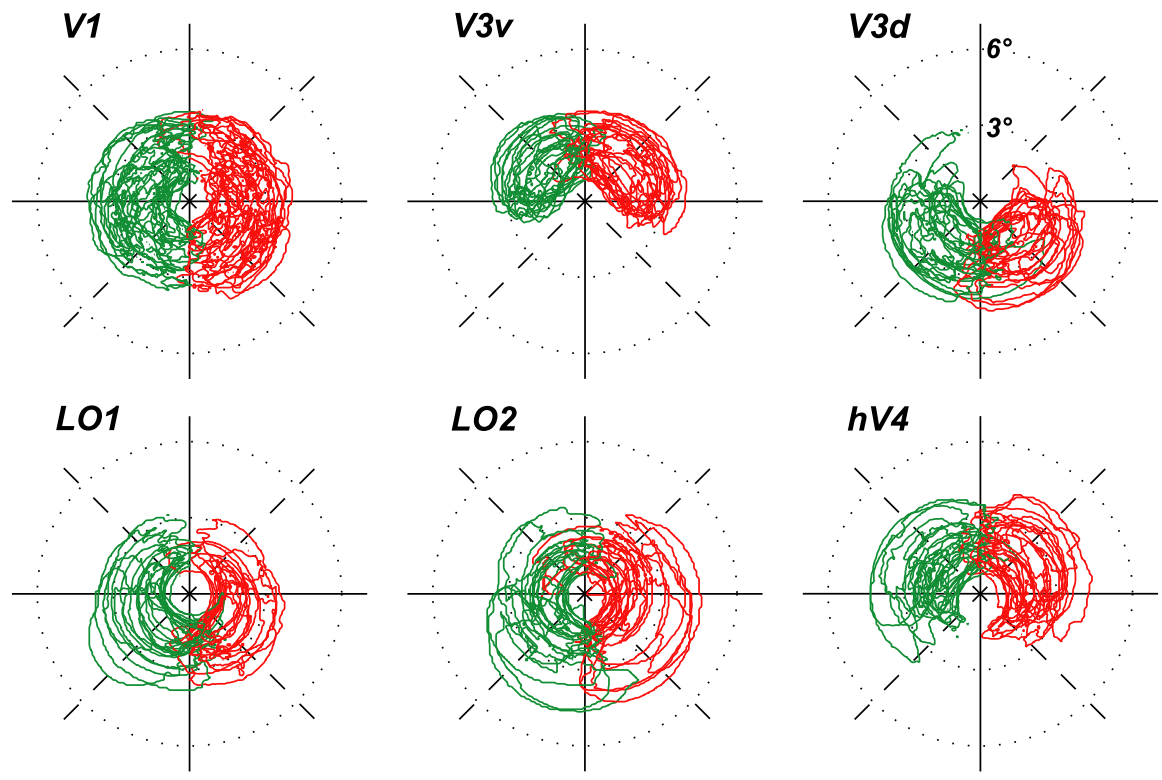

Figure 7. Visual field coverage in L01 and L02 compared with visual areas V3d, V3V, hV4, and V1. Each contour indicates the visual field coverage between 0.5 and $3.5^{\circ}$ eccentricity for an individual subject, estimated from the summed response fields of voxels with coherence $>0.25$. Red and green indicate left and right hemisphere, respectively. Areas V3d and V3v, in each hemisphere, represent the lower and upper contralateral quadrants of the visual field, respectively. In contrast, the entire contralateral hemifield, including both upper and lower quadrants, is represented in each of L01, L02, and V1.

originally described as a representation of the lower contralateral quadrant anterior to the foveal part of V3d (Smith et al., 1998). By this definition, V3B likely corresponds to the posterior half of LO1, which as we have shown represents the entire contralateral hemifield, not just the lower quadrant. Later descriptions of V3B defined it differently, in a more dorsolateral position that does not overlap with either LO1 or LO2 (Press et al., 2001; Wandell et al., 2005). Failing to find a consistent map of polar angle anterior to V3d, Tootell and Hadjikhani (2001) instead proposed an eccentricity-based subdivision of the lateral occipital cortex into two regions, LOC and LOP, representing the central and peripheral visual field, respectively. Our data do not support this parcellation scheme. Instead, LOC and LOP are likely to correspond to the central and peripheral parts, respectively, of both LO1 and LO2. We believe the differences between our results and those of previous studies can be accounted for by improvements in MRI technology, and by differences in experimental design and data analysis methods (for discussion, see supplemental Figures, available at www.jneurosci.org as supplemental material) (Wandell et al., 2005).

Although our data provide compelling evidence for retinotopic organization in the posterior lateral occipital cortex, this organization differed in two important respects from that of earlier retinotopic areas. First, the intersubject variability in the retinotopy of LO1 and LO2 (particularly with respect to the range of polar angles) was greater than for earlier visual areas (although comparable with higher-tier areas such as V7 and VO1). The retinotopy in these areas may be fundamentally more variable and less precise than in early visual areas, analogous to the considerable variability in the location and scatter of receptive fields in higher-tier macaque visual areas such as V4 and TEO (Gattass et al., 1988; Boussaoud et al., 1991). Second, the visual field map in LO2 had two unusual features not observed in other areas: the eccentricity representation in $\mathrm{LO} 2$ exhibited an apparent sudden transition from foveal to peripheral locations in several hemispheres, and the representation of eccentricity differed between the upper and lower quadrants (Figs. 1, 2, supplemental Figs. 2, 4, available at www.jneurosci.org as supplemental material). Additional studies using higherresolution methods will be necessary to determine whether the unusual eccentricity representation in $\mathrm{LO} 2$ can be explained by the high degree of skew in the LO2 map.

\section{Are LO1 and LO2 homologous with macaque V4d and V4t?}

Areas LO1 and LO2 are located between $\mathrm{V} 3 \mathrm{~d}$ and V5/MT+, both of which have well established homologues in the macaque monkey (Kaas and Lyon, 2001; Tootell et al., 2003; Orban et al., 2004). In the most widely used scheme of the macaque visual cortex (Zeki, 1971, 1977; Felleman and Van Essen, 1991), V3d is bordered anteriorly by V4d, anterior to which (and abutting MT) is a small crescentshaped area known as V4t (V4 transitional) or MTc (MT crescent) (Desimone and Ungerleider, 1986; Tootell and Taylor, 1995) (Fig. 3B). Kaas and colleagues (Stepniewska and Kaas, 1996; Stepniewska et al., 2005) have proposed a similar scheme that subdivides this region into a caudal part (DLc) and a rostral part (DLr). Gattass et al. (1988) reported that V4d and V4t each contained a map of the lower visual quadrant, and that these areas were separated by a representation of the horizontal meridian. For eccentricities over $5^{\circ}$, the representation in V4d did not even include the full quadrant; instead, portions of the peripheral lower quadrant were represented in V4v. Similar findings were reported by Desimone and Ungerleider (1986). Recent fMRI measurements of retinotopic organization in macaques (Brewer et al., 2002; Fize et al., 2003) have been mostly consistent with these previous findings, although neither of these fMRI studies found clear evidence for V4t. This discrepancy may reflect the difficulty of resolving such a small area, about $2 \mathrm{~mm}$ in width (Gattass et al., 1988), with fMRI.

Our results suggest that the visual field coverage in this region differs between humans and macaques (Fig. 3), arguing against a direct homology between LO1/LO2 and V4d/V4t. Whereas we found evidence that $\mathrm{LO} 1$ and $\mathrm{LO} 2$ each represent both the upper and lower visual quadrants, the regions of macaque dorsal V4/DL only represent the lower contralateral quadrant, the upper visual field being represented in a complementary ventral map (e.g., for $\mathrm{V} 4 \mathrm{~d}$, the upper field representation is in V4v). Similarly, hV4 (which contains a full hemifield map) is not an exact homolog of macaque $\mathrm{V} 4 \mathrm{v}$ (which contains only the upper visual field representation). In other words, whereas V4d and V4v in the macaque together contain a single complete hemifield map, LO1 and hV4 each contain a full hemifield map and, thus, cannot be the dorsal and ventral parts of a single visual area.

One way to reconcile these interspecies differences in visual field coverage would be to split LO1 and hV4 in the middle along the horizontal meridian representation and consider the resulting posterior parts of the split maps to be the human homologues of monkey V4d and V4v, respectively. However, such a partitioning scheme is highly problematic. First, it would imply that the two anterior 
quadrant representations of LO1 and hV4 would together constitute another area, which has no counterpart in the macaque. Second, the anterior boundary of the resulting human "V4d/V4v" would not coincide with a reversal in the polar angle representation, which is the gold standard for defining boundaries between visual areas retinotopically. Third, we did not find evidence for functional differences between the anterior and posterior halves of either LO1 or hV4 (Fig. 9). Instead, there were functional differences between hV4 and LO1: the increase in response field size with eccentricity was steeper in LO1 than hV4 (Fig. 6B) and the cortical magnification in LO1 differed from that in hV4 (Fig. 5). Furthermore, responses in $\mathrm{LO} 1$ were more strongly modulated by the motion boundary localizer than by the LOC localizer, whereas hV4 was equally strongly modulated by both (Fig. 9D).

\section{Functional specialization of lateral occipital cortex}

LO1 and LO2 are part of the objectselective lateral occipital complex (Malach et al., 1995; Grill-Spector et al., 2001). Several studies have subdivided the LOC based on stimulus selectivities. GrillSpector et al. (1999) and Sawamura et al. (2005) found that the anterior part of the LOC was more invariant to changes in object size and position than the posterior and dorsal part (close to the location of LO1 and LO2). Stanley and Rubin (2005) showed that the LOC could be subdivided into a posterior, lateral part that responded equally well to abstract $2 \mathrm{D}$ shapes and familiar objects, and an anterior and ventral part that responded preferentially to familiar objects. Our results provide additional evidence for a processing hierarchy within the LOC, with increasing selectivity for complex objects and decreased selectivity for low-level features, such as orientation, in higher-tier regions of the LOC. First, we found that LO2 was significantly more responsive than LO1 to objects. Second, whereas LO1 exhibited orientation-selective responses to simple grating stimuli, LO2 showed no selectivity for stimulus orientation. Interestingly, our results suggest a straightforward explanation of the limited positional and scale invariance of the posterior LOC described by Grill-Spector et al. (1999). Because of the retinotopic organization of LO1 and LO2, and the fact that response fields (Fig. 6), although large, only covered a limited region of the visual field, neurons in these areas would not be expected to be invariant to large spatial transformations of their preferred stimuli.

The cortex between dorsal V3 and V5/MT + has also been implicated in the processing of higher-order boundaries, such as motion boundaries (Dupont et al., 1997; Van Oostende et al., 1997, the present study), illusory contours (Mendola et al., 1999), boundaries defined by depth structure (Tyler et al., 2006), and second-order gratings (Larsson et al., 2006). Murray et al. (2003) reported that motion-defined shapes evoked activity in a region
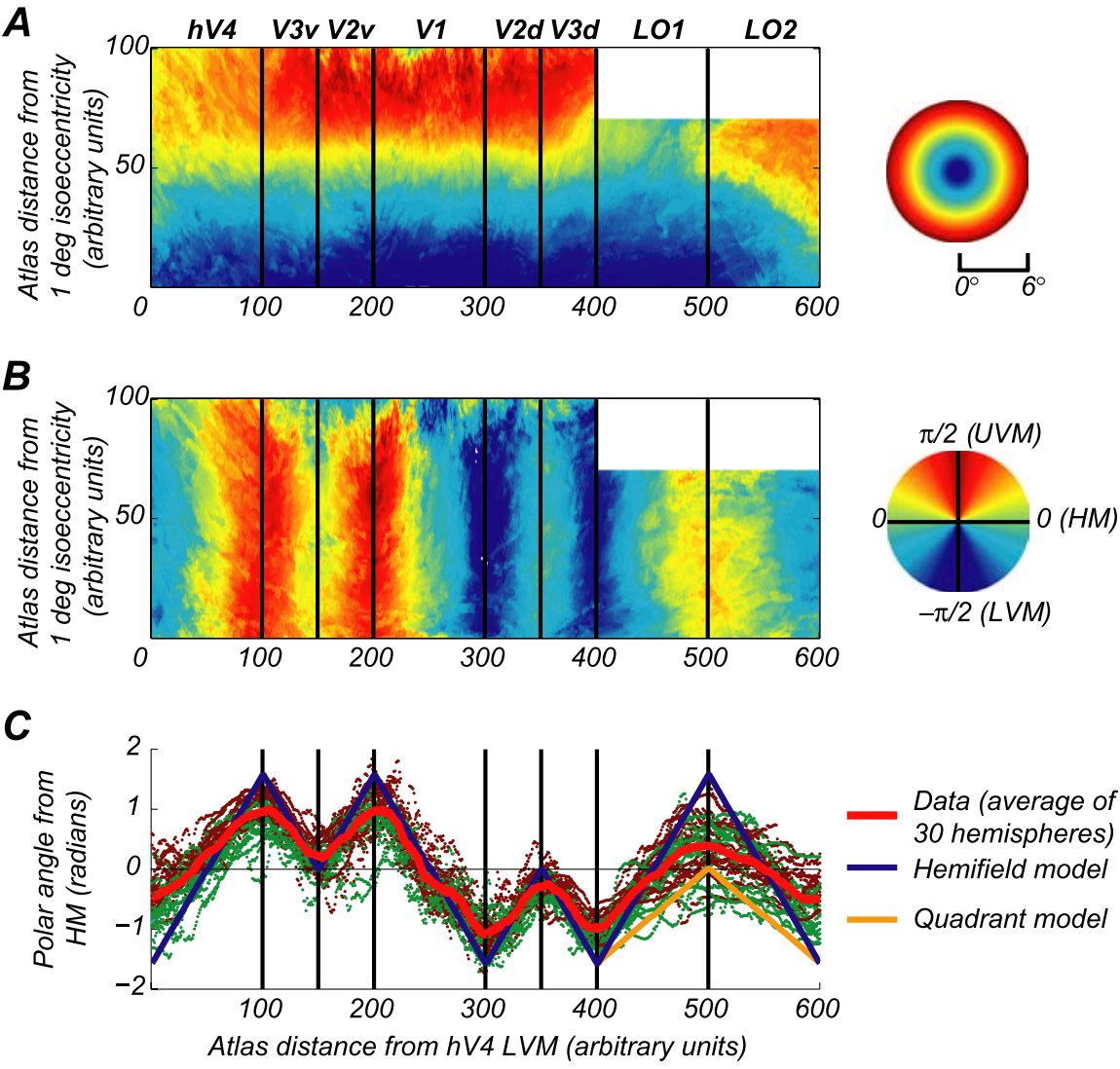

Figure 8. Visual field coverage of retinotopic maps in V1, V2, V3, hV4, L01, and L02. A, Visual field eccentricity (color scale at right) averaged across 30 hemispheres (15 subjects, collapsed across left and right hemispheres) plotted in canonical atlas (a) coordinate system for aligning data across subjects. Data for L01 and L02 restricted to atlas eccentricities $1-4.5^{\circ}$ (see Materials and Methods). B , Visual polar angle (color scale at right) averaged across 30 hemispheres ( 15 subjects). Positive phase angles (red) orange solid curve, predicted polar angle profile assuming only upper quadrant representations in L01 and L02. Note that predictions of the quadrant and hemifield models only differ in L01 and L02.

near the lateral occipital cortex, suggesting a link between responses to motion boundaries and responses to visual shapes, although the location of this region appeared to be closer to V3B or V7 than to LO1/LO2. Zeki et al. (2003) found that cortex between V3d and V5 responded with equal selectivity to shapes defined by color contrast or motion contrast. The responses to such varied types of boundary stimuli in this region suggest that this region may encode higher-order boundaries independent of the cues defining the boundaries.

Others have argued that the LOC, including its posterior part, is primarily responsible for extracting shapes or image regions, not boundaries per se. Tyler et al. (2005a) identified a dorsolateral occipital region anterior to dorsal V3, corresponding to the location of LO1/LO2, that showed enhanced activity to symmetrical patterns. Kourtzi and Kanwisher (2001) found that the LOC responded selectively to perceived shape, but not to the contours that define the shape. Likewise, Stanley and Rubin (2003) found that the LOC responded to perceptually salient regions even 

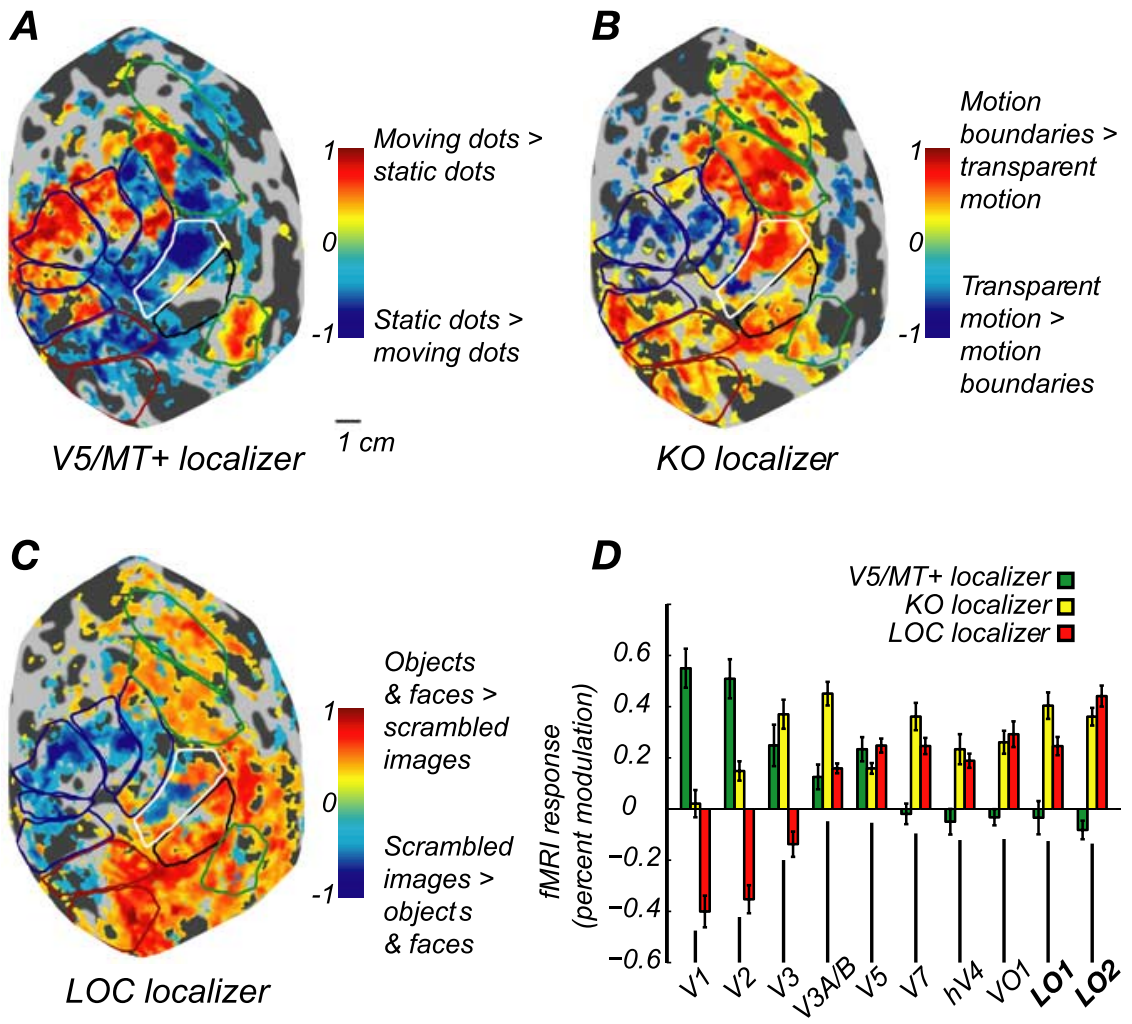

Figure 9. fMRI responses to motion, motion boundaries, and objects. $\boldsymbol{A}-\boldsymbol{C}$, Responses from typical individual subject (S4, right hemisphere). Superimposed visual area regions of interest are color-coded as follows: blue outlines, early visual areas (V1-V3); red outlines, ventral visual areas (hV4, V01); green outlines, dorsal visual areas (V3A/B, V7, V5); white, L01; black, L02. A, fMRI responses to moving random dot patterns alternating with static random dot patterns. Color scale, correlation between $f M R I$ response and a sinusoid with the same (24s) period as the stimulus alternations, for voxels showing strong ( $\mid$ correlation $\mid>0.25$ ) response modulation. Positive correlation indicates stronger fMRI responses to moving dots (test stimulus) than to static dots (control stimulus); negative correlation indicates the opposite response pattern. $\boldsymbol{B}$, fMRI responses to random dot patterns containing motion boundaries alternating with transparently moving random dot patterns. The correlation threshold and color scale are as in $\boldsymbol{A}$. Positive correlation indicates stronger fMRI responses to motion boundary stimuli (test) than to transparent motion (control). C, fMRI responses to images of objects and faces alternating with scrambled versions of the same images. Threshold and color scale are as in $\boldsymbol{A}$. Positive correlation indicates stronger fMRI responses to object and face images (test) than to scrambled images (control). $\boldsymbol{D}$, Amplitudes of mean fMRI responses to localizer stimuli in each visual area, averaged across seven subjects. Positive amplitudes correspond to stronger fMRI responses to test stimuli $(\boldsymbol{A}-\boldsymbol{C}$, red and yellow); negative amplitudes correspond to stronger responses to control stimuli $(\boldsymbol{A}-\boldsymbol{C}$, blue). Error bars indicate SEM across subjects.

when there were no perceived boundaries. Because the two latter studies did not distinguish between different subregions of the LOC, these results are likely to more strongly reflect the response properties of the larger anterior LOC than the properties of the smaller posterior LOC. Together with our finding of boundaryselective responses in LO1 and the limited position and size invariance in the posterior LOC reported by Grill-Spector et al. (1999) and Sawamura et al. (2005), these results imply that, although complex shape information is extracted in the posterior LOC (e.g., LO1 and LO2), invariance to boundary cues and image transformations emerges only in the anterior LOC.

The combination of complex shape selectivity and retinotopic organization in LO1 and LO2 suggests that these areas represent shape information within a spatial coordinate system. This may be useful for a variety of perceptual organization processes that rely on spatial relations in the visual image (e.g., segmentation, grouping, region extraction, boundary extraction, border ownership). The differences in response properties between LO1 and LO2 suggest a segregation of function between the two areas, with LO1 extracting boundary information and LO2 extracting regions and representing shape. In addition, the location of LO1 and LO2, midway between ventral and dorsal visual processing streams, makes these areas well positioned for integrating information from both streams.

\section{References}

Adams DL, Horton JC (2003) A precise retinotopic map of primate striate cortex generated from the representation of angioscotomas. J Neurosci 23:3771-3789.

Bandettini PA, Jesmanowicz A, Wong EC, Hyde JS (1993) Processing strategies for time-course data sets in functional MRI of the human brain. Magn Reson Med 30:161-173.

Boussaoud D, Desimone R, Ungerleider LG (1991) Visual topography of area TEO in the macaque. J Comp Neurol 306:554-575.

Brewer AA, Press WA, Logothetis NK, Wandell BA (2002) Visual areas in macaque cortex measured using functional magnetic resonance imaging. J Neurosci 22:10416-10426.

Brewer AA, Liu J, Wade AR, Wandell BA (2005) Visual field maps and stimulus selectivity in human ventral occipital cortex. Nat Neurosci 8:1102-1109.

Desimone R, Ungerleider LG (1986) Multiple visual areas in the caudal superior temporal sulcus of the macaque. J Comp Neurol 248:164-189.

DeYoe EA, Carman GJ, Bandettini P, Glickman S, Wieser J, Cox R, Miller D, Neitz J (1996) Mapping striate and extrastriate visual areas in human cerebral cortex. Proc Natl Acad Sci USA 93:2382-2386.

Dijkstra EW (1959) A note on two problems in connexion with graphs. Numer Math $1: 269-271$.

Dougherty RF, Press WA, Wandell BA (1999) Perceived speed of colored stimuli. Neuron 24:893-899.

Dougherty RF, Koch VM, Brewer AA, Fischer B, Modersitzki J, Wandell BA (2003) Visual field representations and locations of visual areas V1/ 2/3 in human visual cortex. J Vis 3:586-598.

Duncan RO, Boynton GM (2003) Cortical magnification within human primary visual cortex correlates with acuity thresholds. Neuron 38:659-671.

Dupont P, De Bruyn B, Vandenberghe R, Rosier AM, Michiels J, Marchal G, Mortelmans L, Orban GA (1997) The kinetic occipital region in human visual cortex. Cereb Cortex 7:283-292.

Duvernoy HM (1999) The human brain: surface, blood supply, and threedimensional sectional anatomy. Austria: Springer-Verlag.

Engel SA, Rumelhart DE, Wandell BA, Lee AT, Glover GH, Chichilnisky EJ, Shadlen MN (1994) fMRI of human visual cortex. Nature 369:525.

Engel SA, Glover GH, Wandell BA (1997) Retinotopic organization in human visual cortex and the spatial precision of functional MRI. Cereb Cortex 7:181-192.

Felleman DJ, Van Essen DC (1991) Distributed hierarchical processing in the primate cerebral cortex. Cereb Cortex 1:1-47.

Fisher NI (1993) Statistical analysis of circular data. Cambridge, UK: Cambridge UP.

Fize D, Vanduffel W, Nelissen K, Denys K, Chef d'Hotel C, Faugeras O, Orban GA (2003) The retinotopic organization of primate dorsal V4 and surrounding areas: a functional magnetic resonance imaging study in awake monkeys. J Neurosci 23:7395-7406.

Gattass R, Gross CG, Sandell JH (1981) Visual topography of V2 in the macaque. J Comp Neurol 201:519-539.

Gattass R, Sousa AP, Gross CG (1988) Visuotopic organization and extent of V3 and V4 of the macaque. J Neurosci 8:1831-1845.

Gattass R, Nascimento-Silva S, Soares JG, Lima B, Jansen AK, Diogo AC, Farias MF, Botelho MM, Mariani OS, Azzi J, Fiorani M (2005) Cortical visual areas in monkeys: location, topography, connections, columns, plasticity and cortical dynamics. Philos Trans R Soc Lond B Biol Sci 360:709-731. 
Grill-Spector K, Kushnir T, Edelman S, Itzchak Y, Malach R (1998a) Cueinvariant activation in object-related areas of the human occipital lobe. Neuron 21:191-202.

Grill-Spector K, Kushnir T, Hendler T, Edelman S, Itzchak Y, Malach R (1998b) A sequence of object-processing stages revealed by fMRI in the human occipital lobe. Hum Brain Mapp 6:316-328.

Grill-Spector K, Kushnir T, Edelman S, Avidan G, Itzchak Y, Malach R (1999) Differential processing of objects under various viewing conditions in the human lateral occipital complex. Neuron 24:187-203.

Grill-Spector K, Kourtzi Z, Kanwisher N (2001) The lateral occipital complex and its role in object recognition. Vision Res 41:1409-1422.

Hasson U, Harel M, Levy I, Malach R (2003) Large-scale mirror-symmetry organization of human occipito-temporal object areas. Neuron 37:1027-1041.

Horton JC, Hoyt WF (1991) The representation of the visual field in human striate cortex. A revision of the classic Holmes map. Arch Ophthalmol 109:816-824.

Huk AC, Dougherty RF, Heeger DJ (2002) Retinotopy and functional subdivision of human areas MT and MST. J Neurosci 22:7195-7205.

Jenkinson M, Bannister P, Brady M, Smith S (2002) Improved optimization for the robust and accurate linear registration and motion correction of brain images. NeuroImage 17:825-841.

Kaas JH, Lyon DC (2001) Visual cortex organization in primates: theories of V3 and adjoining visual areas. Prog Brain Res 134:285-295.

Kimmel R, Sethian JA (1998) Computing geodesic paths on manifolds. Proc Natl Acad Sci USA 95:8431-8435.

Kourtzi Z, Kanwisher N (2001) Representation of perceived object shape by the human lateral occipital complex. Science 293:1506-1509.

Kourtzi Z, Erb M, Grodd W, Bulthoff HH (2003) Representation of the perceived 3-D object shape in the human lateral occipital complex. Cereb Cortex 13:911-920.

Larsson J (2001) Imaging vision: functional mapping of intermediate visual processes in man. PhD thesis, Karolinska Institutet, Stockholm, Sweden.

Larsson J, Landy MS, Heeger DJ (2006) Orientation-selective adaptation to first- and second-order patterns in human visual cortex. J Neurophysiol 95:862-881.

Levy I, Hasson U, Avidan G, Hendler T, Malach R (2001) Center-periphery organization of human object areas. Nat Neurosci 4:533-539.

Malach R, Reppas JB, Benson RR, Kwong KK, Jiang H, Kennedy WA, Ledden PJ, Brady TJ, Rosen BR, Tootell RB (1995) Object-related activity revealed by functional magnetic resonance imaging in human occipital cortex. Proc Natl Acad Sci USA 92:8135-8139.

Mendola JD, Dale AM, Fischl B, Liu AK, Tootell RB (1999) The representation of illusory and real contours in human cortical visual areas revealed by functional magnetic resonance imaging. J Neurosci 19:8560-8572.

Murray SO, Olshausen BA, Woods DL (2003) Processing shape, motion and three-dimensional shape-from-motion in the human cortex. Cereb Cortex 13:508-516.

Nestares O, Heeger DJ (2000) Robust multiresolution alignment of MRI brain volumes. Magn Reson Med 43:705-715.

Ogawa S, Lee TM, Kay AR, Tank DW (1990) Brain magnetic resonance imaging with contrast dependent on blood oxygenation. Proc Natl Acad Sci USA 87:9868-9872.

Orban GA, Van Essen D, Vanduffel W (2004) Comparative mapping of higher visual areas in monkeys and humans. Trends Cogn Sci 8:315-324.

Press WA, Brewer AA, Dougherty RF, Wade AR, Wandell BA (2001) Visual areas and spatial summation in human visual cortex. Vision Res 41:1321-1332.

Purdon PL, Weisskoff RM (1998) Effect of temporal autocorrelation due to physiological noise and stimulus paradigm on voxel-level false-positive rates in fMRI. Hum Brain Mapp 6:239-249.

Samaria F, Harter A (1994) Parameterization of a stochastic model for human face identification. Paper presented at Second IEEE Workshop on Applications of Computer Vision, Sarasota, FL.

Sawamura H, Georgieva S, Vogels R, Vanduffel W, Orban GA (2005) Using functional magnetic resonance imaging to assess adaptation and size invariance of shape processing by humans and monkeys. J Neurosci 25:4294-4306.

Schluppeck D, Glimcher P, Heeger DJ (2005) Topographic organization for delayed saccades in human posterior parietal cortex. J Neurophysiol 94:1372-1384.

Sereno MI, Dale AM, Reppas JB, Kwong KK, Belliveau JW, Brady TJ, Rosen BR, Tootell RB (1995) Borders of multiple visual areas in humans revealed by functional magnetic resonance imaging. Science 268:889-893.
Silver MA, Ress D, Heeger DJ (2005) Topographic maps of visual spatial attention in human parietal cortex. J Neurophysiol 94:1358-1371.

Smith AT, Greenlee MW, Singh KD, Kraemer FM, Hennig J (1998) The processing of first- and second-order motion in human visual cortex assessed by functional magnetic resonance imaging (fMRI). J Neurosci 18:3816-3830.

Smith AT, Singh KD, Williams AL, Greenlee MW (2001) Estimating receptive field size from fMRI data in human striate and extrastriate visual cortex. Cereb Cortex 11:1182-1190.

Stanley DA, Rubin N (2003) fMRI activation in response to illusory contours and salient regions in the human lateral occipital complex. Neuron 37:323-331.

Stanley DA, Rubin N (2005) Functionally distinct sub-regions in the lateral occipital complex revealed by fMRI responses to abstract 2-dimensional shapes and familiar objects. J Vis 5:911a.

Stepniewska I, Kaas JH (1996) Topographic patterns of V2 cortical connections in macaque monkeys. J Comp Neurol 371:129-152.

Stepniewska I, Collins CE, Kaas JH (2005) Reappraisal of DL/V4 boundaries based on connectivity patterns of dorsolateral visual cortex in macaques. Cereb Cortex 15:809-822.

Tootell RB, Hadjikhani N (2001) Where is "dorsal V4" in human visual cortex? Retinotopic, topographic and functional evidence. Cereb Cortex 11:298-311.

Tootell RB, Taylor JB (1995) Anatomical evidence for MT and additional cortical visual areas in humans. Cereb Cortex 5:39-55.

Tootell RB, Reppas JB, Kwong KK, Malach R, Born RT, Brady TJ, Rosen BR, Belliveau JW (1995) Functional analysis of human MT and related visual cortical areas using magnetic resonance imaging. J Neurosci 15:3215-3230.

Tootell RB, Mendola JD, Hadjikhani NK, Ledden PJ, Liu AK, Reppas JB, Sereno MI, Dale AM (1997) Functional analysis of V3A and related areas in human visual cortex. J Neurosci 17:7060-7078.

Tootell RB, Hadjikhani NK, Vanduffel W, Liu AK, Mendola JD, Sereno MI, Dale AM (1998) Functional analysis of primary visual cortex (V1) in humans. Proc Natl Acad Sci USA 95:811-817.

Tootell RB, Tsao D, Vanduffel W (2003) Neuroimaging weighs in: humans meet macaques in "primate" visual cortex. J Neurosci 23:3981-3989.

Tsao DY, Freiwald WA, Knutsen TA, Mandeville JB, Tootell RB (2003) Faces and objects in macaque cerebral cortex. Nat Neurosci 6:989-995.

Tyler CW, Baseler HA, Kontsevich LL, Likova LT, Wade AR, Wandell BA (2005a) Predominantly extra-retinotopic cortical response to pattern symmetry. NeuroImage 24:306-314.

Tyler CW, Likova LT, Chen CC, Kontsevich LL, Schira MM, Wade AR (2005b) Extended concepts of occipital retinotopy. Curr Med Imag Rev 1:319-330.

Tyler CW, Likova LT, Kontsevich LL, Wade AR (2006) The specificity of cortical region KO to depth structure. NeuroImage 30:228-238.

Van Essen DC, Newsome WT, Maunsell JH (1984) The visual field representation in striate cortex of the macaque monkey: asymmetries, anisotropies, and individual variability. Vision Res 24:429-448.

Van Oostende S, Sunaert S, Van Hecke P, Marchal G, Orban GA (1997) The kinetic occipital $(\mathrm{KO})$ region in man: an fMRI study. Cereb Cortex 7:690-701.

Wandell BA, Brewer AA, Dougherty RF (2005) Visual field map clusters in human cortex. Philos Trans R Soc Lond B Biol Sci 360:693-707.

Watson JD, Myers R, Frackowiak RS, Hajnal JV, Woods RP, Mazziotta JC, Shipp S, Zeki S (1993) Area V5 of the human brain: evidence from a combined study using positron emission tomography and magnetic resonance imaging. Cereb Cortex 3:79-94.

Yu H, Farley BJ, Jin DZ, Sur M (2005) The coordinated mapping of visual space and response features in visual cortex. Neuron 47:267-280.

Zarahn E, Aguirre GK, D’Esposito M (1997) Empirical analyses of BOLD fMRI statistics. I. Spatially unsmoothed data collected under nullhypothesis conditions. NeuroImage 5:179-197.

Zeki S, Watson JD, Lueck CJ, Friston KJ, Kennard C, Frackowiak RS (1991) A direct demonstration of functional specialization in human visual cortex. J Neurosci 11:641-649.

Zeki S, Perry RJ, Bartels A (2003) The processing of kinetic contours in the brain. Cereb Cortex 13:189-202.

Zeki SM (1971) Convergent input from the striate cortex (area 17) to the cortex of the superior temporal sulcus in the rhesus monkey. Brain Res 28:338-340.

Zeki SM (1977) Colour coding in the superior temporal sulcus of rhesus monkey visual cortex. Proc R Soc Lond B Biol Sci 197:195-223. 